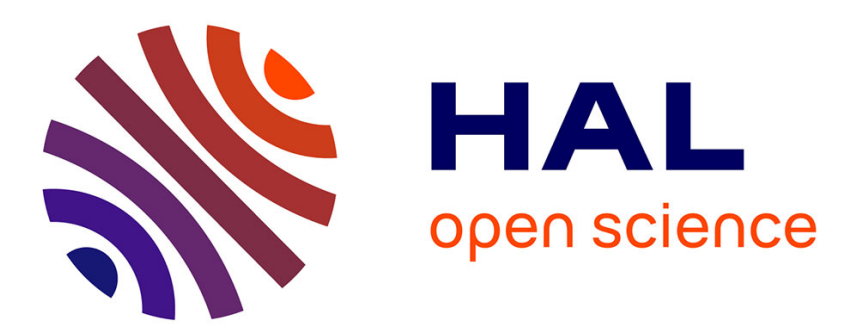

\title{
A purely frequency based Floquet-Hill formulation for the efficient stability computation of periodic solutions of ordinary differential systems
}

Louis Guillot, Arnaud Lazarus, Olivier Thomas, Christophe Vergez, Bruno Cochelin

\section{To cite this version:}

Louis Guillot, Arnaud Lazarus, Olivier Thomas, Christophe Vergez, Bruno Cochelin. A purely frequency based Floquet-Hill formulation for the efficient stability computation of periodic solutions of ordinary differential systems. Journal of Computational Physics, 2020, 416, pp.109477. 10.1016/j.jcp.2020.109477 . hal-02864713

\section{HAL Id: hal-02864713 https://hal.science/hal-02864713}

Submitted on 11 Jun 2020

HAL is a multi-disciplinary open access archive for the deposit and dissemination of scientific research documents, whether they are published or not. The documents may come from teaching and research institutions in France or abroad, or from public or private research centers.
L'archive ouverte pluridisciplinaire HAL, est destinée au dépôt et à la diffusion de documents scientifiques de niveau recherche, publiés ou non, émanant des établissements d'enseignement et de recherche français ou étrangers, des laboratoires publics ou privés. 


\title{
A purely frequency based Floquet-Hill formulation for the efficient stability computation of periodic solutions of ordinary differential systems
}

\author{
Louis Guillot, Arnaud Lazarus, Olivier Thomas, Christophe Vergez, Bruno Cochelin
}

October 9, 2019

\begin{abstract}
Since the founding theory established by G. Floquet more than a hundred years ago, computing the stability of periodic solutions has given rise to various numerical methods, mostly depending on the way the periodic solutions are themselves determined, either in the time domain or in the frequency domain. In this paper, we address the stability analysis of branches of periodic solutions that are computed by combining a pure Harmonic Balance Method (HBM) with an Asymptotic Numerical Method (ANM). HBM is a frequency domain method for determining periodic solutions under the form of Fourier series and ANM is continuation technique that relies on high order Taylor series expansion of the solutions branches with respect to a path parameter. It is well established now that this HBM-ANM combination is efficient and reliable, provided that the system of ODE is first of all recasted with quadratic nonlinearities, allowing an easy manipulation of both the Taylor and the Fourier series. In this context, Hill's method, a frequency domain version of Floquet theory, is revisited so as to become a by-product of the HBM applied to a quadratic system, allowing the stability analysis to be implemented in an elegant way and with good computing performances. The different types of stability changes of periodic solutions are all explored and illustrated through several academic examples, including systems that are autonomous or not, conservative or not, free or forced.
\end{abstract}

\section{Introduction}

Periodic stationary states of dynamical systems arise in many places in applied mathematics from physics and engineering to biology and chemistry or even economics [GH83b, Str01]. Examples include, to mention just a few: the nonlinear vibrations of guitar strings [TVK00, TLT10] or microelectromechanical resonators $\left[\mathrm{KHP}^{+}\right.$09, LTD12], the stationary notes of clarinets [KVC12], the rotating state of bacterial flagella [GGHW00] or the modeling of business cycles [Goo51]. Depending on the nature, complexity or size of the discrete dynamical systems under study, several numerical approaches are possible to compute periodic stationary solutions [AP98]. For a given set of initial conditions, one could always directly integrate in time the set of nonlinear Ordinary Differential Equations (ODEs) until an eventual periodic state is reached. Doing so, transient and stationary motions are accessible but those methods are time consuming and the computed states are critically dependent on the chosen initial conditions [AP98, TSD16]. A more elaborate approach would necessitate additional shooting methods [NB95, Sey88], that, on top of direct timeintegration, consist in using iterative solvers or standard minimization algorithms to find only the initial conditions associated with periodic stationary states. Spectral methods are another possibility that requires no time integration and consists in decomposing the sought periodic orbit in an orthogonal basis of functions. Amongst them, orthogonal collocation [Doe81, DKK91], based on periodic polynomial representation functions, and 
harmonic balance [Ura65, KB16, CV09], based on Fourier series, are the most used methods in the time and frequency domain, respectively. The goal of spectral methods is to transform the original set of ODEs in a larger set of nonlinear algebraic equations. One main advantage of the spectral approaches is that nonlinear algebraic equations are a very relevant framework to use continuation techniques based on predictor-corrector algorithms [Kel87, DKK91] or asymptotic numerical methods [Coc94, CDPF94a, CDPF94b] that allow to follow all the bifurcated stationary states of a dynamical system with respect to relevant control parameters.

A crucial feature, especially when navigating in complex bifurcation diagrams, is to assess the local stability of the computed periodic orbits, since dynamical systems only eventually evolve towards solutions that are asymptotically stable [Kha96]. The linear stability of periodic stationary states of nonlinear dynamical systems can be determined thanks to Floquet theory [Flo79, WW96] applied to the Linear Time-Periodic System (LTPS) governing the dynamical perturbation around a periodic stationary state. Two main numerical methods exist that naturally emerge whether the periodic stationary state has been discretized in the time or frequency domain. In the time domain, the computation of the monodromy or transfer matrix of the LTPS over one period allows for the determination of Floquet multipliers whose absolute value reveal the local stability of the studied state [NB95, WH01]. In the frequency domain, the Fourier expansion of the LTPS allows, through harmonic balancing, for the construction of the Hill matrix whose eigenvalues, also known as Floquet exponents, determine the stability and the nature of the bifurcations of the periodic solutions [Hil86, ZHA04, BL18]. On one hand, the monodromy matrix usually appears as a by-product of the shooting $\left[\mathrm{PVS}^{+} 09\right]$ or orthogonal collocation methods [DKK91] and the stability analysis is therefore efficiently integrated in the computation of periodic solutions in the time domain. On the other hand however, the computation of periodic states of nonlinear systems and the determination of their stability are, in most cases [Moo05], two disconnected steps in the frequency domain [LT10, PBTJR13, DRMK15].

In order to improve the general efficiency of nonlinear algorithms such as continuation techniques in the frequency domain, we investigated whether Hill's method could be automatically integrated in the harmonic balance formalism for the computation of periodic solutions of ordinary differential systems. The first part of this paper reports how Hill's matrix could be constructed as a by-product of the harmonic balance method (HBM) applied to compute the periodic solutions of a set of quadratic ordinary differential equations, which has been shown to be a very efficient and convenient framework for continuation techniques based on HBM and Asymptotic Numerical Methods (ANM) [CDPF94b, CV09, GCV19]. By implementing our theoretical derivations in MANLAB [ACV05, $\mathrm{KAL}^{+}$10], the matlab package for interactive continuation and bifurcation analysis of nonlinear ODEs based on harmonic balance and quadratic recast, we illustrate in a second part the feasibility and relevance of our work through the numerical computation of the periodic solutions of some archetypal dynamical systems.

\section{Floquet-Hill's method for quadratic systems}

In this section, a quadratic recast of the equations is used for an efficient and automatic computation of Hill's matrix. First, Floquet's theory is recalled to be applied to a quadratic recast of the equations. Then, the Harmonic Balance Method (HBM) is recalled and used on the quadratic linearized system to compute Hill's matrix. Finally, the implementation of an automatic algorithm is discussed. 


\subsection{Floquet theory}

Let us consider the following system of ordinary differential equations:

$$
\dot{\boldsymbol{x}}(t)=\boldsymbol{f}(\boldsymbol{x}(t), t, \lambda)
$$

with $\boldsymbol{x}(t) \in \mathbb{R}^{N}$ the state vector, $t \in \mathbb{R}$ the time, $\lambda \in \mathbb{R}$ a control parameter, $f: \mathbb{R}^{N} \times \mathbb{R} \rightarrow$ $\mathbb{R}^{N}$ an analytic function periodic in its second argument and $N \in \mathbb{N}^{*}$ the size of the dynamical system. $\boldsymbol{f}$ may explicitly depend on $t$ (the system is non-autonomous) or not (the system is autonomous). In the following, the dependence in $\lambda$ will be ommited when not necessary to lighten the writing.

Let $t \mapsto \boldsymbol{x}_{0}(t)$ be a $T$-periodic solution of system (1) for a particular value $\lambda_{0}$ of $\lambda$, with $T \in \mathbb{R}$ its minimal period. The linear stability of this periodic solution $\boldsymbol{x}_{0}(t)$ can be studied by superimposing a small disturbance $\boldsymbol{y}$ such that $\boldsymbol{x}(t)=\boldsymbol{x}_{0}(t)+\boldsymbol{y}(t)$, that verifies the linearized system:

$$
\dot{\boldsymbol{y}}(t)=\boldsymbol{J}(t) \boldsymbol{y}(t)
$$

where $\boldsymbol{J}(t)$ is the $N \times N$ Jacobian matrix of $\boldsymbol{f}$ computed at $\boldsymbol{X}=\boldsymbol{x}_{0}$ and $\lambda=\lambda_{0}$ :

$$
\boldsymbol{J}(t)=\frac{\partial \boldsymbol{f}}{\partial \boldsymbol{x}}\left(x_{0}(t), t, \lambda_{0}\right) .
$$

It has to be noticed that $t \mapsto J(t)$ is $T$-periodic. This allows, thanks to Floquet's theorem (see e.g. [NB95]), to write the perturbation $\boldsymbol{y}(t)$ as:

$$
\boldsymbol{y}(t)=\sum_{n=1}^{N} c_{n} \boldsymbol{y}_{n}(t),
$$

where the fundamental solutions $\boldsymbol{y}_{n}(t), n=1, \ldots, N$ of system (2) can be written under the so-called Floquet form:

$$
\boldsymbol{y}_{n}(t)=\mathrm{e}^{\alpha_{n} t} \boldsymbol{p}_{n}(t),
$$

where $\alpha_{n}, n=1, \ldots, N$ are complex numbers called the Floquet exponents and $\boldsymbol{p}_{n}$ are $T$-periodic $N$-dimensional periodic vectors. Considering (4) and (5), the real parts of the Floquet exponents $\alpha_{n}$ give the stability of the periodic solution $\boldsymbol{x}_{0}$. If they are all negative, it is asymptotically stable and if one of them is positive, it is locally unstable.

\subsection{Floquet theory on a quadratic system}

Following the works of [CV09, KCV13, GCV19], the nonlinear function $\boldsymbol{f}$ of equations (1) is written in a quadratic form with the use of $N_{a}$ auxiliary variables, denoted by $\boldsymbol{x}_{a}(t) \in \mathbb{R}^{N_{a}}$. The dynamical system (1) is thus rewritten:

$$
\left\{\begin{array}{c}
\dot{\boldsymbol{x}}=\boldsymbol{g}\left(\boldsymbol{x}, \boldsymbol{x}_{a}, t\right) \\
0=\boldsymbol{g}_{a}\left(\boldsymbol{x}, \boldsymbol{x}_{a}, t\right)
\end{array}\right.
$$

where the vector functions $\boldsymbol{g}$ and $\boldsymbol{g}_{a}$ are of size $N$ and $N_{a}$ respectively and the size of the full system is $N_{f}=N+N_{a}$. In this augmented system, the second vector equation (6b) of size $N_{a}$ fully defines the $N_{a}$ additional variables $\boldsymbol{x}_{a}$ as a function of the primary variables $\boldsymbol{x}$ (this definition can be implicit, in the form of Eq. (6b), or explicit, this equation being rewritten $\boldsymbol{x}_{a}=\tilde{g}_{a}(\boldsymbol{x}, t)$ ). Then, the first equation (6a) is equivalent to the initial dynamical system (1). 
We consider here that $\boldsymbol{g}$ and $\boldsymbol{g}_{a}$ are quadratic in $\boldsymbol{x}$ and $\boldsymbol{x}_{a}$ and periodic in $t$. These properties are written:

$$
\left\{\begin{aligned}
\boldsymbol{g}\left(\boldsymbol{x}_{f}, t\right) & =\boldsymbol{c}(t)+\boldsymbol{l}\left(\boldsymbol{x}_{f}\right)+\boldsymbol{q}\left(\boldsymbol{x}_{f}, \boldsymbol{x}_{f}\right), \\
\boldsymbol{g}_{a}\left(\boldsymbol{x}_{f}, t\right) & =\boldsymbol{c}_{a}(t)+\boldsymbol{l}_{a}\left(\boldsymbol{x}_{f}\right)+\boldsymbol{q}_{a}\left(\boldsymbol{x}_{f}, \boldsymbol{x}_{f}\right),
\end{aligned}\right.
$$

where $\boldsymbol{x}_{f}(t) \in \mathbb{R}^{N_{f}}$ is the augmented state vector:

$$
\boldsymbol{x}_{f}=\left[\begin{array}{c}
\boldsymbol{x} \\
\boldsymbol{x}_{a}
\end{array}\right]=\left[\boldsymbol{x} ; \boldsymbol{x}_{a}\right]
$$

where the notation ";" means that the vectors are gathered row-wise. $\boldsymbol{c}(t) \in \mathbb{R}^{N}, \boldsymbol{c}_{a}(t) \in$ $\mathbb{R}^{N_{a}}$ are $T$-periodic vectors, $\boldsymbol{l}: \mathbb{R}^{N_{f}} \rightarrow \mathbb{R}^{N}, \boldsymbol{l}_{a}: \mathbb{R}^{N_{f}} \rightarrow \mathbb{R}^{N_{a}}$ are linear operators and $\boldsymbol{q}: \mathbb{R}^{N_{f}} \times \mathbb{R}^{N_{f}} \rightarrow \mathbb{R}^{N}, \boldsymbol{q}_{a}: \mathbb{R}^{N_{f}} \times \mathbb{R}^{N_{f}} \rightarrow \mathbb{R}^{N_{a}}$ are bilinear operators, not necessary symmetric. We consider here that the explicit time dependence is concentrated in the constant operators $\boldsymbol{c}$ and $\boldsymbol{c}_{a}$, without loss of generality, since additional variables in $\boldsymbol{x}_{a}$ can be defined as time functions. As an example, the case of parametrically excited systems will be treated in section 3.3.2.

Let us define $\boldsymbol{x}_{f 0}=\left[\boldsymbol{x}_{0} ; \boldsymbol{x}_{a 0}\right]$ a $T$-periodic solution of the full quadratic system (6). Let $\boldsymbol{y}_{f}(t)$ be a small perturbation of $\boldsymbol{x}_{f 0}$, such that $\boldsymbol{x}_{f}=\boldsymbol{x}_{f 0}+\boldsymbol{y}_{f}$. Replacing this expression in Eq (6), expanding the result in powers of $\boldsymbol{y}_{f}$ and keeping only linear terms in $\boldsymbol{y}_{f}=\left[\boldsymbol{y} ; \boldsymbol{y}_{a}\right]$ gives:

$$
\left\{\begin{array}{c}
\dot{\boldsymbol{y}}=\frac{\partial \boldsymbol{g}}{\partial \boldsymbol{x}} \boldsymbol{y}+\frac{\partial \boldsymbol{g}}{\partial \boldsymbol{x}_{a}} \boldsymbol{y}_{a} \\
0=\frac{\partial \boldsymbol{g}_{a}}{\partial \boldsymbol{x}} \boldsymbol{y}+\frac{\partial \boldsymbol{g}_{a}}{\partial \boldsymbol{x}_{a}} \boldsymbol{y}_{a}
\end{array}\right.
$$

where the four Jacobian matrices of $\boldsymbol{g}$ and $\boldsymbol{g}_{a}$ are taken at $\boldsymbol{x}_{f}=\boldsymbol{x}_{f 0}\left(\boldsymbol{x}=\boldsymbol{x}_{0}, \boldsymbol{x}_{a}=\boldsymbol{x}_{a 0}\right)$. With the help of Eq. (7), this set of equations can also be written:

$$
\left\{\begin{array}{l}
\dot{\boldsymbol{y}}=\boldsymbol{l}\left(\boldsymbol{y}_{f}\right)+\boldsymbol{q}\left(\boldsymbol{x}_{f 0}, \boldsymbol{y}_{f}\right)+\boldsymbol{q}\left(\boldsymbol{y}_{f}, \boldsymbol{x}_{f 0}\right), \\
0=\boldsymbol{l}_{a}\left(\boldsymbol{y}_{f 0}\right)+\boldsymbol{q}_{a}\left(\boldsymbol{x}_{f 0}, \boldsymbol{y}_{f}\right)+\boldsymbol{q}_{a}\left(\boldsymbol{y}_{f}, \boldsymbol{x}_{f 0}\right) .
\end{array}\right.
$$

The above equations show that the Jacobian matrices of Eqs. (9) are linear functions of $\boldsymbol{x}_{0}(t), \boldsymbol{x}_{a 0}(t)$, because of the quadratic expression of $\boldsymbol{g}$ and $\boldsymbol{g}_{a}$. They do not depend on the constant operators $\boldsymbol{c}(t)$ and $\boldsymbol{c}_{a}(t)$ and they are function of time only through the $T$-periodic functions $\boldsymbol{x}_{0}(t), \boldsymbol{x}_{a 0}(t)$, and not explicitely. They are thus $T$-periodic in time.

If the auxiliary variables $\boldsymbol{x}_{a}$ are defined with a linear declaration rule in Eq. (6b) (see [GCV19] for all the details), the matrix $\partial \boldsymbol{g}_{a} / \partial \boldsymbol{x}_{a}$ is non-singular and Eq. (9b) gives

$$
\boldsymbol{y}_{a}=-\left(\left[\frac{\partial \boldsymbol{g}_{a}}{\partial \boldsymbol{x}_{a}}\right]^{-1} \frac{\partial \boldsymbol{g}_{a}}{\partial \boldsymbol{x}}\right) \boldsymbol{y} .
$$

Replacing the above expression in Eq. (9a) leads to write:

$$
\dot{\boldsymbol{y}}(t)=\underbrace{\left(\frac{\partial \boldsymbol{g}}{\partial \boldsymbol{x}}(t)-\frac{\partial \boldsymbol{g}}{\partial \boldsymbol{x}_{a}}(t)\left[\frac{\partial \boldsymbol{g}_{a}}{\partial \boldsymbol{x}_{a}}(t)\right]^{-1} \frac{\partial \boldsymbol{g}_{a}}{\partial \boldsymbol{x}}(t)\right)}_{\boldsymbol{J}(t)} \boldsymbol{y}(t)
$$

Comparing the above expression with the original linearized system (2) shows that it is possible to exactly compute the Jacobian matrix $\boldsymbol{J}(t)$ with the Jacobian matrices of the augmented system vector functions $\boldsymbol{g}$ and $\boldsymbol{g}_{a}$. It is then possible to compute Floquet exponents $\alpha_{n}$ and Floquet eigenfunctions $\boldsymbol{p}_{n}$ as explained at the end of section 2.1. 


\subsection{Harmonic Balance Method and Hill's Method}

An efficient method for computing a periodic solution $\boldsymbol{x}_{0}(t)$ of the smooth dynamical system (1) is to look for the solution in the form of a truncated Fourier series. This is the so-called Harmonic Balance Method, abbreviated HBM here. Then, it is natural to estimate the stability of the solution $\boldsymbol{x}_{0}(t)$ by computing the Floquet exponents in the Frequency domain, leading to the so-called Hill's method. Some previous works [LT10] already used a similar framework with a quadratic format of the equations to compute Hill's matrix efficiently. We propose in the following a generalization of this method.

\subsubsection{Quick recall of the Harmonic Balance Method}

The HBM consists in expanding the $T$-periodic unknown solution $\boldsymbol{x}(t)$ of system (1) in a truncated Fourier series:

$$
\boldsymbol{x}(t)=\sum_{h=-H}^{H} \boldsymbol{x}^{(h)} \mathrm{e}^{i h \omega t}=\boldsymbol{x}^{(0)}+\sum_{h=1}^{H}\left(\boldsymbol{x}_{c}^{(h)} \cos (h \omega t)+\boldsymbol{x}_{s}^{(h)} \sin (h \omega t)\right),
$$

where the angular frequency is $\omega=2 \pi / T$. In the following, bold capital letters will denote the vectors gathering all Fourier components of a particular $T$-periodic function. For instance, the Fourier coefficients of $\boldsymbol{x}(t)$ will be denoted by $\boldsymbol{X}$, which can be either a vector of the complex Fourier coefficient $\left[\boldsymbol{x}^{(-H)} ; \ldots ; \boldsymbol{x}^{(0)} ; \ldots, \boldsymbol{x}^{(H)}\right]$ or the vector of the real Fourier coefficients $\left[\boldsymbol{x}^{(0)} ; \boldsymbol{x}_{c}^{(1)} ; \ldots ; \boldsymbol{x}_{c}^{(H)} ; \boldsymbol{x}_{s}^{(1)} ; \ldots, \boldsymbol{x}_{s}^{(H)}\right]$, without loss of generality, since both complex and real Fourier series can be equally used. The size of this vector is $(2 H+1)$ times the size of $\boldsymbol{x}$.

The system of equations (6) is then written in the frequency domain by expanding it on the real Fourier basis of order $H$ [CV09]. The equivalence between the real form and the complex form of the Fourier series allows to switch between the two, using the one that is the most appropriate for our purpose. The article [GVVC17] explains in detail how to use efficiently the complex representation to compute the products with a convolution and how to recover real operators from complex operators.

The system (6) written in the frequency domain formally looks like:

$$
\left\{\begin{aligned}
\omega \boldsymbol{D}(\boldsymbol{X}) & =\boldsymbol{G}\left(\boldsymbol{X}, \boldsymbol{X}_{a}\right), \\
0 & =\boldsymbol{G}_{a}\left(\boldsymbol{X}, \boldsymbol{X}_{a}\right),
\end{aligned}\right.
$$

with

$$
\left\{\begin{array}{c}
\boldsymbol{G}\left(\boldsymbol{X}, \boldsymbol{X}_{a}\right)=\boldsymbol{C}+\boldsymbol{L}\left(\boldsymbol{X}_{f}\right)+\boldsymbol{Q}\left(\boldsymbol{X}_{f}, \boldsymbol{X}_{f}\right) \\
\boldsymbol{G}_{a}\left(\boldsymbol{X}, \boldsymbol{X}_{a}\right)=\boldsymbol{C}_{a}+\boldsymbol{L}_{a}\left(\boldsymbol{X}_{f}\right)+\boldsymbol{Q}_{a}\left(\boldsymbol{X}_{f}, \boldsymbol{X}_{f}\right) .
\end{array}\right.
$$

In the above system, $\boldsymbol{X}$ is of size $N(2 H+1), \boldsymbol{X}_{a}$, is of size $N_{a}(2 H+1)$ and $\boldsymbol{X}_{f}=\left[\boldsymbol{X} ; \boldsymbol{X}_{a}\right]$ is of size $N_{f}(2 H+1)$ with $N_{f}=N+N_{a}$. They gather the Fourier coefficients of $\boldsymbol{x}, \boldsymbol{x}_{a}$ and $\boldsymbol{x}_{f} . \omega \boldsymbol{D}$ is a linear operator in $\boldsymbol{X}$ of size $N(2 H+1)$, equivalent to the time differentiation in the frequency domain; $\boldsymbol{C}, \boldsymbol{L}$ and $\boldsymbol{Q}$ are respectively constant, linear and quadratic operators in $\boldsymbol{X}_{f}$, of size $N(2 H+1)$, corresponding to Eq. (6a) in the Frequency domain; $\boldsymbol{C}_{a}, \boldsymbol{L}_{a}$ and $\boldsymbol{Q}_{a}$ are respectively constant, linear and quadratic operators in $\boldsymbol{X}_{f}$, of size $N_{a}(2 H+1)$, corresponding to Eq. (6b) in the Frequency domain. All the operators are constructed from the operators $\boldsymbol{g}$ and $\boldsymbol{g}_{a}$ of the ODE system as described in [CV09]. The full algebraic system (14) truncated at order $H$ is of size $(2 H+1) N_{f}$.

\subsubsection{Hill's method on a quadratic system}

Hill's method is basically a frequency domain approach of Floquet theory. The idea is to solve Eq. (2) or equivalently Eq. (12) in the frequency domain by expanding the Jacobian matrix $\boldsymbol{J}(t)$ in Fourier series. 
Equations (9) are now rewritten using Floquet theory. Eqs. (4) and (5) show that $\boldsymbol{y}(t)$ is a linear combination of the Floquet forms $\boldsymbol{y}_{n}(t)$. Since Eq. (9) is linear, it is sufficient to verify it with any fundamental solution $\boldsymbol{y}_{n}(t)$, written here without the $n$ subscript to lighten the notations: $\boldsymbol{y}(t)=\mathrm{e}^{\alpha t} \boldsymbol{p}(t)$ with $\boldsymbol{p}(t)$ being $T$-periodic. Consequently to Eq. (11), we can write $\boldsymbol{y}_{a}(t)=\mathrm{e}^{\alpha t} \boldsymbol{p}_{a}(t)$, with the same Floquet exponent $\alpha$ and $\boldsymbol{p}_{a}(t)$ being $T$-periodic. We also denote $\boldsymbol{p}_{f}(t)=\left[\boldsymbol{p}(t) ; \boldsymbol{p}_{a}(t)\right]$. Substituting those two Floquet forms into Eqs. (9), one obtains:

$$
\left\{\begin{aligned}
\dot{\boldsymbol{p}}+\alpha \boldsymbol{p} & =\frac{\partial \boldsymbol{g}}{\partial \boldsymbol{x}} \boldsymbol{p}+\frac{\partial \boldsymbol{g}}{\partial \boldsymbol{x}_{a}} \boldsymbol{p}_{a}, \\
0 & =\frac{\partial \boldsymbol{g}_{a}}{\partial \boldsymbol{x}} \boldsymbol{p}+\frac{\partial \boldsymbol{g}_{a}}{\partial \boldsymbol{x}_{a}} \boldsymbol{p}_{a} .
\end{aligned}\right.
$$

The above system is now written in the frequency domain; using Eqs. (14) and (15), one obtains:

$$
\left\{\begin{aligned}
\omega \boldsymbol{D}(\boldsymbol{P})+\alpha \boldsymbol{P} & =\boldsymbol{L}\left(\boldsymbol{P}_{f}\right)+\boldsymbol{Q}\left(\boldsymbol{X}_{f 0}, \boldsymbol{P}_{f}\right)+\boldsymbol{Q}\left(\boldsymbol{P}_{f}, \boldsymbol{X}_{f 0}\right), \\
0 & =\boldsymbol{L}_{a}\left(\boldsymbol{P}_{f}\right)+\boldsymbol{Q}_{a}\left(\boldsymbol{X}_{f 0}, \boldsymbol{P}_{f}\right)+\boldsymbol{Q}_{a}\left(\boldsymbol{P}_{f}, \boldsymbol{X}_{f 0}\right) .
\end{aligned}\right.
$$

where $\boldsymbol{P}$ is of size $N(2 H+1), \boldsymbol{P}_{a}$, is of size $N_{a}(2 H+1)$ and $\boldsymbol{P}_{f}=\left[\boldsymbol{P} ; \boldsymbol{P}_{a}\right]$ is of size $N_{f}(2 H+1)$ with $N_{f}=N+N_{a}$. They gather the Fourier coefficients of $\boldsymbol{p}(t), \boldsymbol{p}_{a}(t)$ and $\boldsymbol{p}_{f}(t)$. The above equations, linear in $\boldsymbol{P}$ and $\boldsymbol{P}_{a}$, can be written:

$$
\left\{\begin{aligned}
\omega \boldsymbol{D}(\boldsymbol{P})+\alpha \boldsymbol{P} & =\frac{\partial \boldsymbol{G}}{\partial \boldsymbol{X}} \boldsymbol{P}+\frac{\partial \boldsymbol{G}}{\partial \boldsymbol{X}_{a}} \boldsymbol{P}_{a} \\
0 & =\frac{\partial \boldsymbol{G}_{a}}{\partial \boldsymbol{X}} \boldsymbol{P}+\frac{\partial \boldsymbol{G}_{a}}{\partial \boldsymbol{X}_{a}} \boldsymbol{P}_{a} .
\end{aligned}\right.
$$

The second set of the above equations allows to write $\boldsymbol{P}_{a}$ as a function of $\boldsymbol{P}$ in the same manner as in the system (11):

$$
\boldsymbol{P}_{a}=-\left[\frac{\partial \boldsymbol{G}_{a}}{\partial \boldsymbol{X}_{a}}\right]^{-1} \frac{\partial \boldsymbol{G}_{a}}{\partial \boldsymbol{X}} \boldsymbol{P} .
$$

Replacing $\boldsymbol{P}_{a}$ by its expression in Eq. (18a) gives the following eigenvalue problem:

$$
\left(\boldsymbol{H}-s \boldsymbol{I}_{N(2 H+1)}\right) \boldsymbol{Q}=\mathbf{0},
$$

which is the system (12) projected on the Floquet forms in the frequency domain, where the so-called Hill matrix is:

$$
\boldsymbol{H}=\frac{\partial \boldsymbol{G}}{\partial \boldsymbol{X}}-\frac{\partial \boldsymbol{G}}{\partial \boldsymbol{X}_{a}}\left[\frac{\partial \boldsymbol{G}_{a}}{\partial \boldsymbol{X}_{a}}\right]^{-1} \frac{\partial \boldsymbol{G}_{a}}{\partial \boldsymbol{X}}-\omega \boldsymbol{D}
$$

In the above Eq. (20), the eigenvalue and eigenvectors have been denoted by $(s, \boldsymbol{Q})$ instead of $(\alpha, \boldsymbol{P})$ for a reason that will be addressed in the next section.

As compared to the previous work [LT10] on the subject, the Hill matrix can be automatically computed by knowing only the quadratic operators $\boldsymbol{g}$ and $\boldsymbol{g}_{a}$ (and their extension in the frequency domain, $\boldsymbol{G}$ and $\boldsymbol{G}_{a}$ ), using the condensation of the auxiliary variables that has been explained in [GCV19]. A numerical comparison of this automatic construction of Hill's matrix and a construction by hand, using the method described in [BL18], has been done and the results are consistent once the order of truncation is strictly more than 1 . 

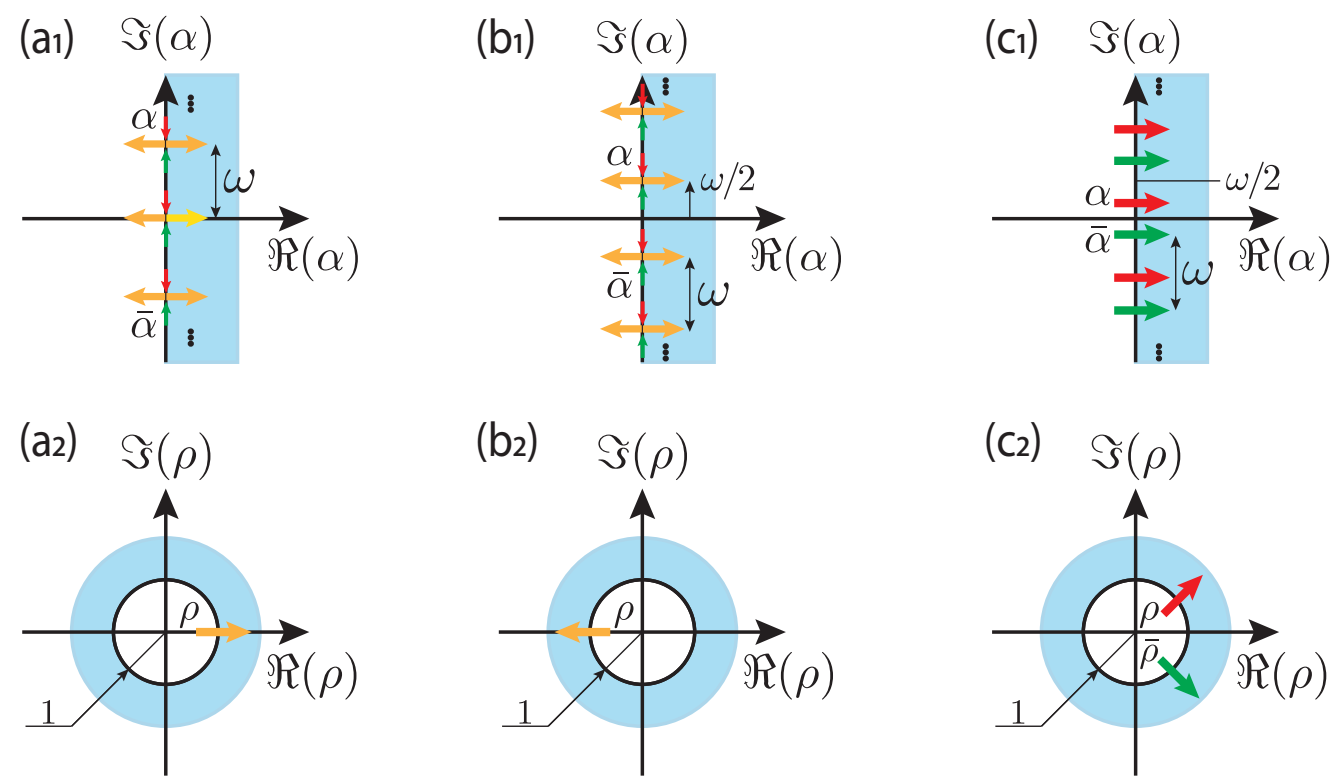

Figure 1: Bifurcation and local stability analysis of a periodic solution by studying the locus of Floquet exponents $\alpha_{n}$ (first row) and multipliers $\rho_{n}$ (second row) in the complex plane. (a) Simple bifurcation (saddle-node, pitchfork). (b) Flip or period doubling bifurcation. (c) Secondary Hopf or Neimark-Sacker bifurcation.

\subsection{Floquet exponents and Hill's eigenvectors}

Computing the spectrum of the square Hill matrix $\boldsymbol{H}$ of size $N(2 H+1)$ (Eq. (20)), we get $N(2 H+1)$ eigenvalues $s_{l}$ and complex eigenvectors $\boldsymbol{Q}_{l}$ of size $N(2 H+1), l=1, \ldots, N(2 H+$ 1 ). When the number of harmonics of the HBM tends to infinity, at the limit $H \rightarrow+\infty$, the eigenvalues are actually not all independent [ZHA04, Moo05, LTD12, BL18] since they verify the relation:

$$
s_{l}=s_{n}^{(h)}=\alpha_{n}+i h \omega,
$$

with $l=1, \ldots, N(2 H+1), n=1, \ldots, N, h=-H, \ldots-1,0,1, \ldots, H$. An analogous property holds for the eigenvectors. If the complex Fourier expansion of the periodic vector $\boldsymbol{p}_{n}(t)$ is written:

$$
\boldsymbol{p}_{n}(t)=\sum_{h=-H}^{H} \boldsymbol{p}_{n}^{(h)} \mathrm{e}^{i h \omega t}
$$

we gather its Fourier components in the vector:

$$
\boldsymbol{P}_{n}=\left[\boldsymbol{p}_{n}^{(-H)} ; \ldots ; \boldsymbol{p}_{n}^{(-1)} ; \boldsymbol{p}_{n}^{(0)} ; \boldsymbol{p}_{n}^{(1)} ; \ldots ; \boldsymbol{p}_{n}^{(H)}\right]
$$

of size $N(2 H+1)$. Then, the $l$-th. eigenvector $\boldsymbol{Q}_{l}$ of Eq. (20) can be written:

$$
\boldsymbol{Q}_{l}=\boldsymbol{Q}_{n}^{(h)}=\left[\boldsymbol{p}_{n}^{(-H+h)} ; \ldots ; \boldsymbol{p}_{n}^{(-1+h)} ; \boldsymbol{p}_{n}^{(0+h)} ; \boldsymbol{p}_{n}^{(1+h)} ; \ldots ; \boldsymbol{p}_{n}^{(H+h)}\right]
$$

Observing Eqs. (22) and (25) shows that each Floquet mode $\left(\alpha_{n}, \boldsymbol{p}_{n}(t)\right), n=1, \ldots N$ is computed redundantly $(2 H+1)$ times by the Hill eigenproblem $(20)$. In the case of the eigenvalues, a given $\alpha_{n}$ can be theoretically computed equally $(2 H+1)$ times with $\alpha_{n}=s_{n}^{(h)}-i h \omega$. A graphical representation of this property is shown in the complex plane in the first row of Fig. 1 in which the $(2 H+1)$ eigenvalues $s_{n}^{(h)}$ associated to $\alpha_{n}$, for a given $n$, appear with the same real part translated vertically $(2 H+1)$ times of a quantity $\omega$. For the eigenvectors, Eq. (25) shows that $\boldsymbol{Q}_{n}^{(h)}$ contains $\boldsymbol{P}_{n}$ with its Fourier components translated from a factor $h$, so that it is obtained $2 H+1$ times. This is illustrated in 

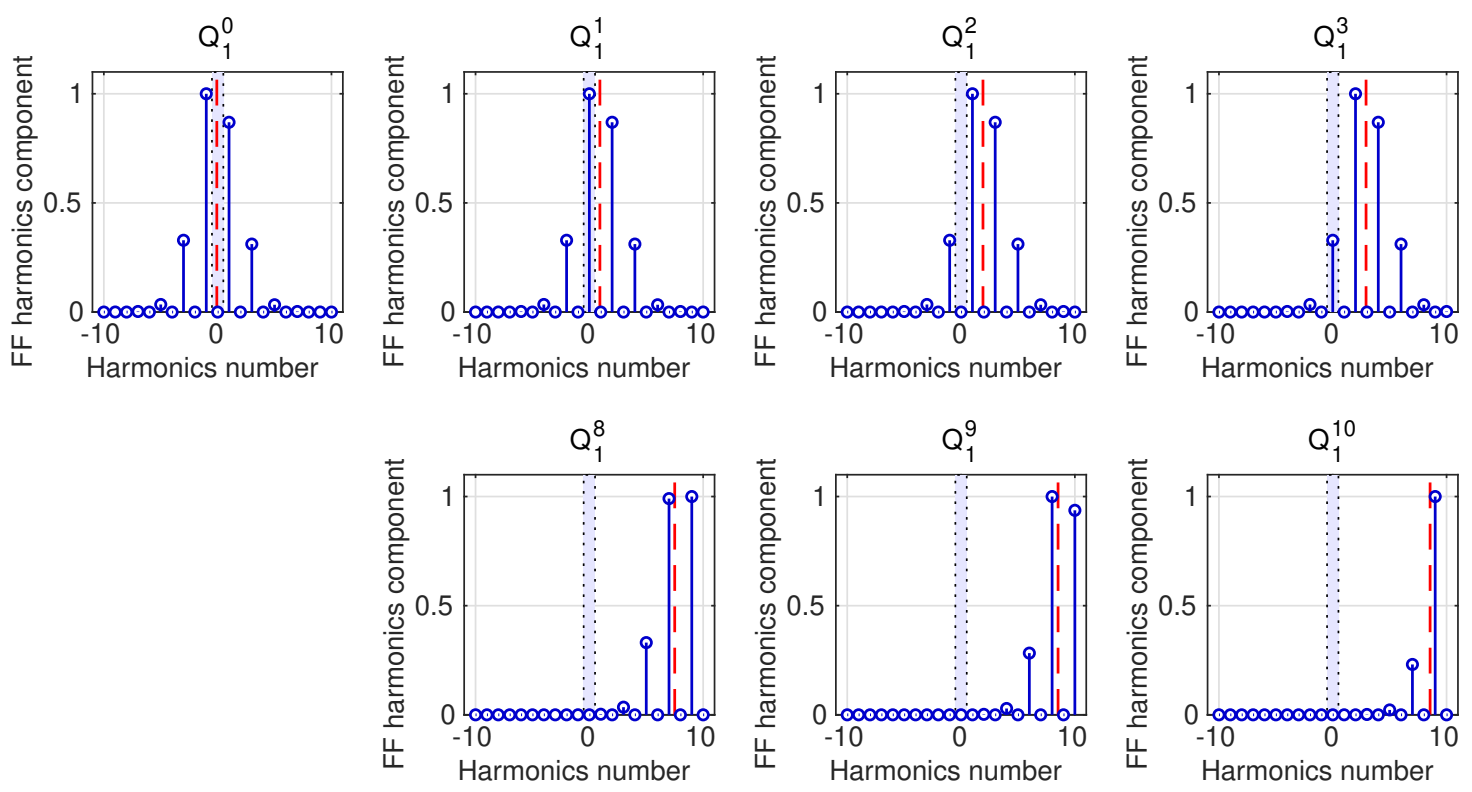

Figure 2: Plot of the components of some eigenvectors $\boldsymbol{Q}_{n}^{(h)}$ of Eq. (21). For a particular point close to the primary resonance of the forced Duffing oscillator (Fig. 6), computed with $H=10$ harmonics, the eigenvectors $\boldsymbol{Q}_{1}^{(h)}$ linked to the first Floquet form $(n=1)$ are shown. Each plot shows the components of $\boldsymbol{Q}_{1}^{(h)}$ for a particular value of $h, h \in 0,1,2,3,8,9,10$. The components of $\boldsymbol{Q}_{1}^{(h)}$ (Eq. 25) are shown on the y-axis as the norm $\left\|\boldsymbol{p}_{1}^{(i)}\right\|$ as a function of $i=-H, \ldots 0 \ldots, H$ in the x-axis.

Fig. 2 in which $\boldsymbol{Q}_{1}^{(h)}$ has the same pattern than $\boldsymbol{Q}_{1}^{(0)}$, in term of Fourier components, but translated of $h$ harmonics.

The above redundancy results holds only at the limit $H \rightarrow+\infty$. In practice, $H$ is finite and the redundancy is not exact. Consequently, some eigensolutions $\left(s_{n}^{(h)}, \boldsymbol{Q}_{n}^{(h)}\right)$ give a better estimate of $\left(\alpha_{n}, \boldsymbol{P}_{n}\right)$, for a given $n$, and there is a necessity to sort the spectrum of the truncated Hill's matrix in order to properly compute the basis of $N$ converged independent Floquet forms among the $N(2 H+1)$ computed eigensolutions. Two main sorting methods exist in the literature whether the sorting algorithm is based on the eigenvalues relation Eq (22) [ZHA04] or the eigenvectors equation (25) [LT10, BL18]. In the following, we use the latter class of sorting algorithms since literature has shown it was the fastest to converge [BL18].

Before describing the eigenvector sorting algorithm, Fig. 2 illustrates the loss of exact redundancy of the eigenvectors when $H$ is finite. One can first notice that the property of Eq. (25) holds only for low values (close to zero) of $|h|^{1}$. In particular, for $h=1,2,3$, the pattern of $\boldsymbol{Q}_{1}^{(0)}$ is exactly recovered on $\boldsymbol{Q}_{1}^{(h)}$ but translated of $h$ harmonics on the right (it would be on the left for $h<0)$. However, for high values of $|h|$, close to $H(h=8,9,10)$, the pattern is distorted, illustrating that $\boldsymbol{Q}_{1}^{(8)}, \boldsymbol{Q}_{1}^{(9)}$ and $\boldsymbol{Q}_{1}^{(10)}$ in this case are bad estimates of $\boldsymbol{P}_{1} \simeq \boldsymbol{Q}_{1}^{(0)}$. Considering that the Fourier expansion of $\boldsymbol{p}_{n}(t)$ is convergent, its amplitude is concentrated in its low harmonics and its high harmonics are zero; $\boldsymbol{Q}_{1}^{(0)}$ in Fig. 2 then seems the better estimate of $\boldsymbol{P}_{n}$. Consequently, the $N$ better estimates of the $\boldsymbol{P}_{n}, n=1, \ldots N$, are the eigenvectors $\boldsymbol{Q}_{l}, l=1, \ldots N(2 H+1)$ with the most centered pattern. A quantitative

\footnotetext{
${ }^{1}$ For a sake of conciseness, Fig. 2 shows $\boldsymbol{Q}_{1}^{(h)}$ for positive values of $h$ only. The same behaviour has been observed for negative values of $h$
} 
indicator of this is the weighted mean of $\boldsymbol{Q}_{l}$, defined by:

$$
w_{l}=\sum_{i=-H}^{H} h\left\|\boldsymbol{p}_{l}^{(i)}\right\| / \sum_{i=-H}^{H}\left\|\boldsymbol{p}_{l}^{(i)}\right\|,
$$

where $\|\bullet\|$ is the norm of $\bullet$ and $\boldsymbol{p}_{l}^{(i)}$ is defined in Eq. (25). The $N$ most converged eigenvectors are then the $N$ ones with a weighted mean $w_{l}$ the closest to zero. Moreover, apart when $h$ is close to $H$, the particular form of Eq (25) implies $w_{l}=w_{n}+h$ for $h=-H, \ldots,-1,0,1, \ldots, H$ so the $N$ fundamental eigenvalues $\alpha_{n}$ and eigenvectors $\boldsymbol{P}_{n}$ are finally the ones for which the weighted means $w_{n}$ are inside the primitive cell $-1 / 2 \leq w_{n}<$ $1 / 2$. Upon convergence, i.e. for a sufficiently large $H$, only $N$ weighted means should be found in the primitive cell. This property is illustrated in Fig. 2, in which $w_{l}$ is shown in a red vertical dashed line and the primitive cell in light blue shading. Only $\boldsymbol{Q}_{1}^{(0)}$ has its weighted mean inside the primitive cell. Currently, there is no rigorous mathematical proof on the convergence of this sorting method but the literature [LT10, TLT10, LTD12, MTTB14, GVVC17, BL18, Laz19], and now the following results, demonstrate to date the great efficiency of the method.

Using the above algorithm, it is possible to estimate the stability of the periodic solution $\boldsymbol{x}_{0}(t)$ by either observing the locus of the $N$ Floquet exponents $\alpha_{n}(n=1, \ldots, N)$ with respect to the imaginary axis in the complex plane, or more classically (see e.g. [NB95]) the locus of the $N$ Floquet multipliers, defined by $\rho_{n}=\exp \left(\alpha_{n} T\right)$ with $T=2 \pi / \omega$, with respect to the unit circle (see Fig. 1). A crossing of those limits implies a change of stability and the nature of this crossing is linked to a particular type of bifurcation. Moreover, like classical normal modes for linear systems of ODEs with constant coefficients, Floquet forms constitute an orthogonal basis for linear time-periodic systems [CW84, BL18]. In particular, in the case of a Neimark-Sacker bifurcation, the quasi-periodic function $\boldsymbol{p}_{n}(t) e^{\alpha_{n} t}$ at the onset of instability gives the direction of the new quasi-periodic solution. This practical use of Floquet forms will be illustrated later on in Section 3.2.2. Note that the modal information $\boldsymbol{p}_{n}(t)$ is almost never used in the literature for navigation in bifurcation diagrams, although it could be used for initial guess to compute new bifurcation branches.

All the developments of section 2.3 are valid for either real coefficients or complex coefficients for the Fourier expansion (see Eq. (13)). Consequently, if real coefficients are chosen to compute the periodic solution $\boldsymbol{x}_{0}(t)$ (which is the case for the computation shown in this article), the Hill matrix (21) will also be composed of real coefficients, as well as the computed eigenvectors $\boldsymbol{Q}_{l}$. On the other hand, the sorting of the Hill eigenvectors explained in the present section has to be done on the complex Fourier coefficients. As a consequence, the real coefficients of $\boldsymbol{Q}_{l}$ have to be transformed into their complex counterpart before using the sorting algorithm.

\section{Validation on some test cases}

In this section, we have implemented the aforementioned theoretical developments in the Matlab continuation software MANLAB that is based on the asymptotic numerical expansion of quadratic systems of algebraic equations obtained by the harmonic balance method $\left[\mathrm{man}, \mathrm{KAL}^{+} 10\right]$. To validate and challenge our new stability algorithm, we review here the most archetypal bifurcation diagrams of periodic solutions of classical nonlinear dynamical systems, with an emphasis on the stability determination.

\subsection{Continuation algorithm}

The continuation algorithm used to draw the bifurcation diagrams of all the systems studied is based on a Taylor series expansion of the solution branch coupled with the harmonic 
balance method. It is freely available online on a dedicated website [man] and has been the subject of many publications, hence the method is not recalled here. The stability computation using Hill's method as described in the previous sections has been implemented within the framework of this tool and is then completely automatized once the system to solve is written in the appropriate format. The examples below are recast in a quadratic format following the works [GCV19, GVC18, KCV13] and the previous sections.

The general setting to have the stability computation automatically available is to recast the system of Ordinary Differential Equations in the appropriate format:

$$
\left\{\begin{array}{l}
0=\boldsymbol{g}\left(\boldsymbol{x}, \boldsymbol{x}_{a}, t\right)-\dot{\boldsymbol{x}} \\
0=\boldsymbol{g}_{a}\left(\boldsymbol{x}, \boldsymbol{x}_{a}, t\right)
\end{array}\right.
$$

which is almost exactly the same as (6), although the derivatives appear with a -1 sign. The stability analysis is performed at the starting and the ending point of a continuation step. If the system encounters a bifurcation, it automatically searches for the exact bifurcation point using a dichotomy algorithm.

Depending on the choice of the bifurcation parameter and the kind of dynamical system, three cases can be considered in practice. To precise this, the algebraic system (14) solved by the continuation method is formally written:

$$
\boldsymbol{R}\left(\boldsymbol{X}_{f}, \lambda, \omega\right)=\mathbf{0},
$$

which has $N_{f}(2 H+1)$ equations (the size of $\left.\boldsymbol{R}\right)$ and $N_{f}(2 H+1)+2$ unknowns. The implicit functions theorem states that with $N_{f}(2 H+1)+1$ unknowns, this system can be solved. In practice, either an additional phase equation is needed, or one of the parameters $\lambda$ or $\omega$ has to be prescribed, leading to the three following cases:

- (i) autonomous non conservative systems: in this case, a phase equation is added to (28), imposing that for the $i$-th. unknown $\dot{x}_{i}(t=0)=0$, for a given $i$. This case is considered in section 3.2 ;

- (ii) autonomous conservative systems: in this case, as explained in section 3.4, both a fictitious bifurcation parameter $\lambda$ and a phase equation are added. The computation of nonlinear modes of conservative systems is in this case [RKC16];

- (iii) forced systems: in this case, no phase condition is needed since the phase of the periodic solution is imposed by the forcing and is thus a consequence of the computation. Usually, one considers $\omega$ as the bifurcation parameter $(\lambda=\omega)$. Another case can be to prescribe $\omega$ at a fixed value and to leave $\lambda$ free, as the forcing amplitude. Some examples are given in section 3.3.

\subsection{Autonomous non conservative system}

We consider here some autonomous dynamical systems that lead to self-sustained oscillations. They encounter typical bifurcations such as saddle-node, pitchfork and NeimarkSacker bifurcations associated to changes of stability. Moreover, since the systems are autonomous, one of the Floquet multiplier is always equal to $\rho_{1}=1$. For practical reasons, in the implemented algorithm, it is first identified among all the $\rho_{n}, n=1, \ldots N$ by the one the closest to $1\left(\rho_{1}=\min _{n}\left|\rho_{n}-1\right|\right)$. Then, $\rho_{1}$ is not considered for the stability tests. It is thus not plotted on the Floquet multiplier plots of this section. 

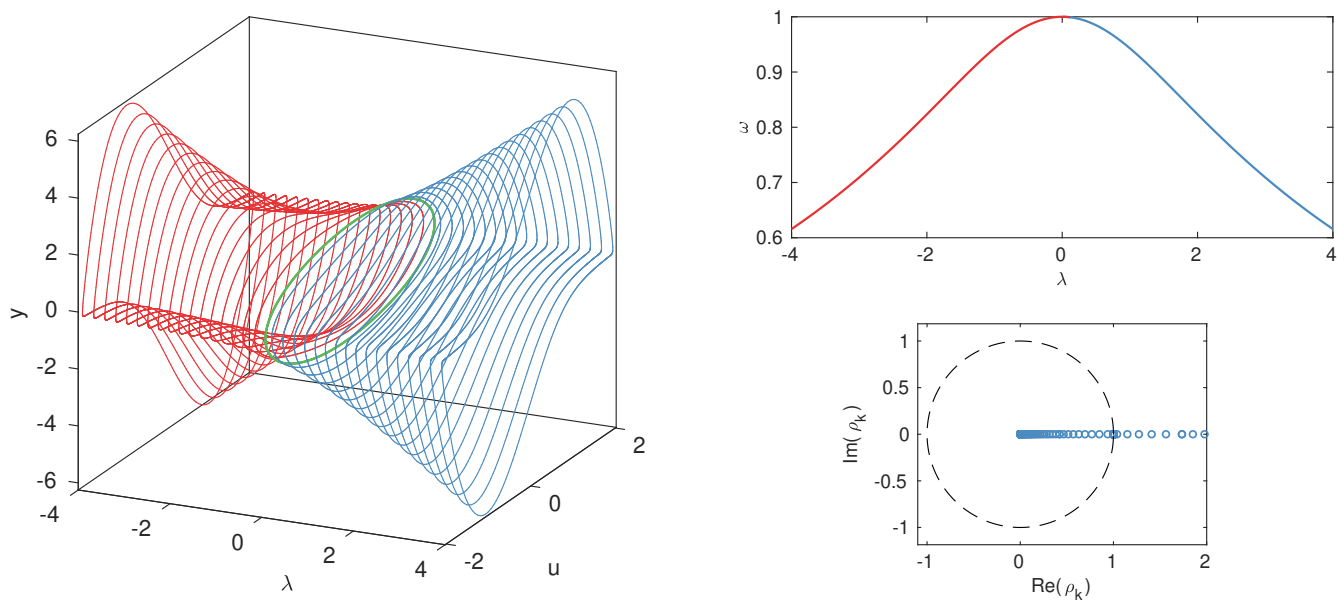

Figure 3: Bifurcation diagram (in the form of a waterfall of the phase diagrams), evolution of the periodic solution frequency $\omega$ as a function of the bifurcation parameter $\lambda$ and evolution of the Floquet multipliers of the Van der Pol oscillator (29). The solution-branch has been computed with $H=50$ harmonics. Stable solutions are represented in blue, unstable solutions in red. A global bifurcation occurs for $\lambda=0$ since (29) degenerates into a conservative harmonic oscillator whose periodic orbit is shown in green.

\subsubsection{Van der Pol oscillator}

Let us consider a classical Van der Pol oscillator

$$
\ddot{x}-\lambda\left(1-x^{2}\right) \dot{x}+x=0
$$

Introducing $y=\dot{x}, r=1-x^{2}$ and $v=\lambda y$ yields the following quadratic system:

$$
\begin{array}{ll}
0=y & -\dot{x} \\
0=r v-x & -\dot{y} \\
0=r-\left(1-x^{2}\right) & \\
0=v-\lambda y &
\end{array}
$$

This system has a branch of periodic solutions existing for all the values of $\lambda$ that encounters a global bifurcation for $\lambda=0$. Indeed for $\lambda=0$ fixed, equation (29) describes a harmonic oscillator and thus a branch of periodic solutions of a conservative system. As explained before, one of the Floquet multiplier is equal to one (not represented in right figure 3) and the other one is real greater than 1 if $\lambda<0$ (the solution is unstable) and between 0 and 1 if $\lambda>0$ (the solution is stable).

\subsubsection{Coupled Van der Pol oscillators}

Let us consider a system of coupled Van der Pol oscillators similar to the one studied in [GVVC17] :

$$
\begin{array}{ll}
\ddot{x}_{1}+a_{1} \dot{x}_{1}+x_{1} & =\lambda\left(\dot{x}_{1}+\dot{x}_{2}\right)\left(1+b\left(x_{1}+x_{2}\right)+c\left(x_{1}+x_{2}\right)^{2}\right) \\
\ddot{x}_{2}+a_{2} \dot{x}_{2}+\Omega^{2} x_{2} & =2 \lambda\left(\dot{x}_{1}+\dot{x}_{2}\right)\left(1+b\left(x_{1}+x_{2}\right)+c\left(x_{1}+x_{2}\right)^{2}\right)
\end{array}
$$

where $x_{1}$ and $x_{2}$ are the two physical variables of the system and $\lambda$ is the control parameter. Introducing $z_{1}=\dot{x}_{1}, z_{2}=\dot{x}_{2}, r=\left(x_{1}+x_{2}\right)^{2}, w=\left(x_{1}+x_{2}\right)\left(z_{1}+z_{2}\right)$ and $v=r\left(z_{1}+z_{2}\right)$ 
yields the following quadratic system:

$$
\begin{array}{ll}
0=z_{1} & -\dot{x}_{1} \\
0=\lambda\left(z_{1}+z_{2}\right)+b \lambda w+c \lambda v-x_{1}-a_{1} z_{1} & -\dot{z}_{1} \\
0=z_{2} & -\dot{x}_{2} \\
0=2 \lambda\left(z_{1}+z_{2}\right)+2 b \lambda w+2 c \lambda v-\Omega^{2} x_{2}-a_{2} z_{2} & -\dot{z}_{2} \\
0=r-\left(x_{1}+x_{2}\right)^{2} & \\
0=w-\left(x_{1}+x_{2}\right)\left(z_{1}+z_{2}\right) & \\
0=v-r\left(z_{1}+z_{2}\right) &
\end{array}
$$
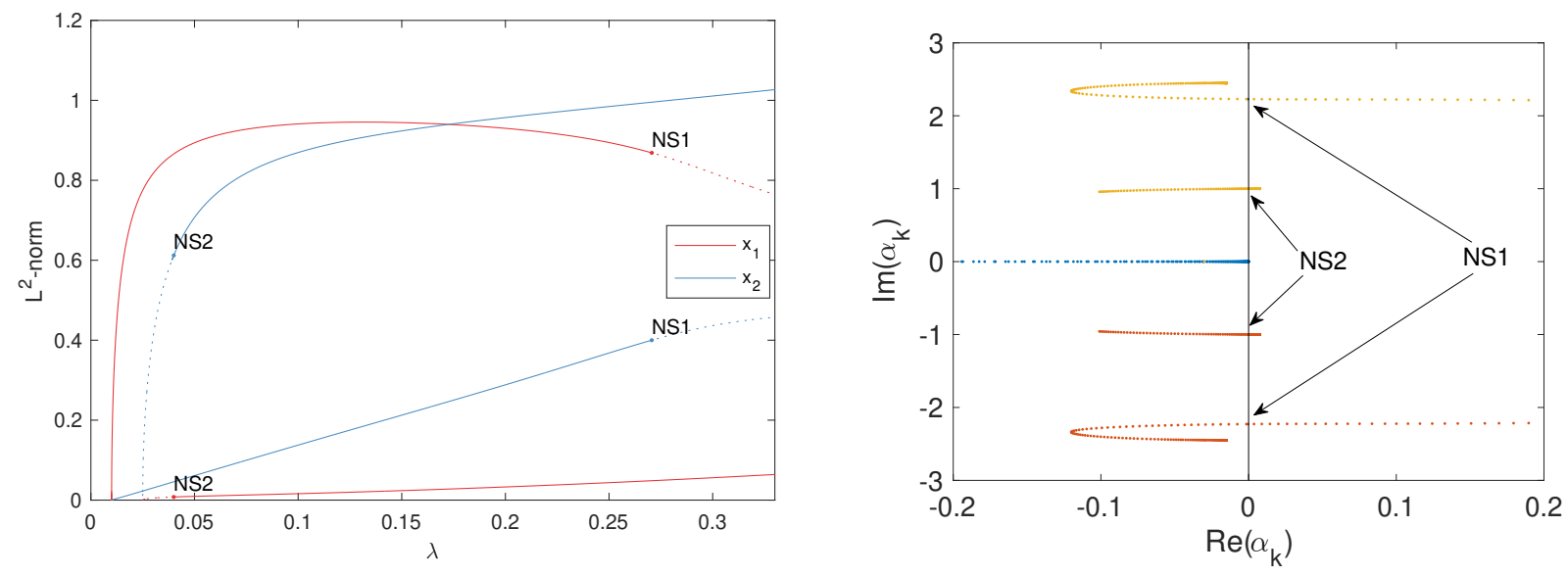

Figure 4: Bifurcation diagram and evolution of the Floquet multipliers around the bifurcations of Eqs. (31). The solution branches have been computed with $H=30$ harmonics. Stable solutions are represented in solid lines, unstable solutions in dotted lines. The two degrees of freedom $x_{1}$ and $x_{2}$ are represented on both the branches that arise from the two Hopf bifurcations. The Floquet exponents are represented around the two Neimark-Sacker bifurations NS1 and NS2 that occur on the first and the second branch respectively.

The chosen parameters are: $a_{1}=0.01, a_{2}=0.05, b=c=-2, \Omega=\sqrt{6}$. This system encounters two Hopf bifurcations for $\lambda=a_{1}$ and $2 \lambda=a_{2}$, in our case for $\lambda=0.01$ and $\lambda=0.025$. From these two bifurcations, periodic branches arise that both encounter a Neimark-Sacker bifurcation. Figure 4 shows the bifurcation diagram of the system on the left-hand-side and the Floquet exponents on the right-hand-side. The first NeimarkSacker bifurcation NS1 occurs on the branch arising from the first Hopf bifurcation. The solutions on this branch are approximately $2 \pi$-periodic, the dynamics is governed by the first degree of freedom $x_{1}$. Thus, the new angular frequency that appear at NS1 is expected to be around $\Omega$, the eigen angular frequency of the second oscillator. On the figure, the imaginary part of the Floquet exponents that crosses the imaginary axis, i.e. the angular frequency introduced in the system, is around 2.2 that is close to $\Omega=\sqrt{6}=2.45$. In the same manner, at the second Neimark-Sacker bifurcation NS2 the angular frequency introduced in the system is around 1, which is the eigen angular frequency of the first oscillator.

At the bifurcation NS1, the shape of the arising quasi-periodic (QP) solution can be approximated using the Floquet forms. If $\boldsymbol{x}_{0}(t)=\left[x_{1}(t) ; x_{2}(t)\right]$ denotes the state vector of periodic physical solutions with fundamental frequency $\omega$, the perturbed solution is given by $\boldsymbol{x}_{F F}(t)=\boldsymbol{x}_{0}(t)+A \boldsymbol{y}_{n}(t)$ where $\boldsymbol{y}_{n}(t)=\mathrm{e}^{\alpha_{n} t} \boldsymbol{p}_{n}(t)$ given in (5) is a Floquet form of $\boldsymbol{x}_{0}(t)$ and $A$ is a complex constant that can be chosen freely. We recall the Floquet exponents $\alpha_{n}$ and periodic eigenfunctions $\boldsymbol{p}_{n}(t)$, with period $T=2 \pi / \omega$, are obtained by solving the automatically computed Hill eigenvalue problem as explained in section 2 . A good approximate of the bifurcated quasi-periodic solution at NS1 is to choose the 
quasi-periodic Floquet form $\boldsymbol{y}_{n}(t)$ associated with $\Re\left(\alpha_{n}\right) \rightarrow 0$ that is responsible for the bifurcation and a relatively small constant $A$ for the linear approximation to be correct. Figure 5 shows the expected shape of QP solution $\boldsymbol{x}_{F F}(t)$ in the time domain obtained for $A=0.05$ and the QP solution, slightly after NS1, computed with a QP harmonic balance method that has been described in [GVVC17]. The two solutions are in a qualitatively good agreement. It seems that there is a small phase shift between the two shapes but as these are QP solutions, it is not so easy to state for sure. As it makes it easier to compare the signal shapes, the author decided not to find a better phase agreement. This approach based on Floquet forms leads to an easier initialization of QP orbits after Neimark-Sacker bifurcations which is known to be a very tough problem when dealing with QP signals. The possibility to initialize the computation of QP solution branches using this technique is implemented in the version of MANLAB available online, used for this article.

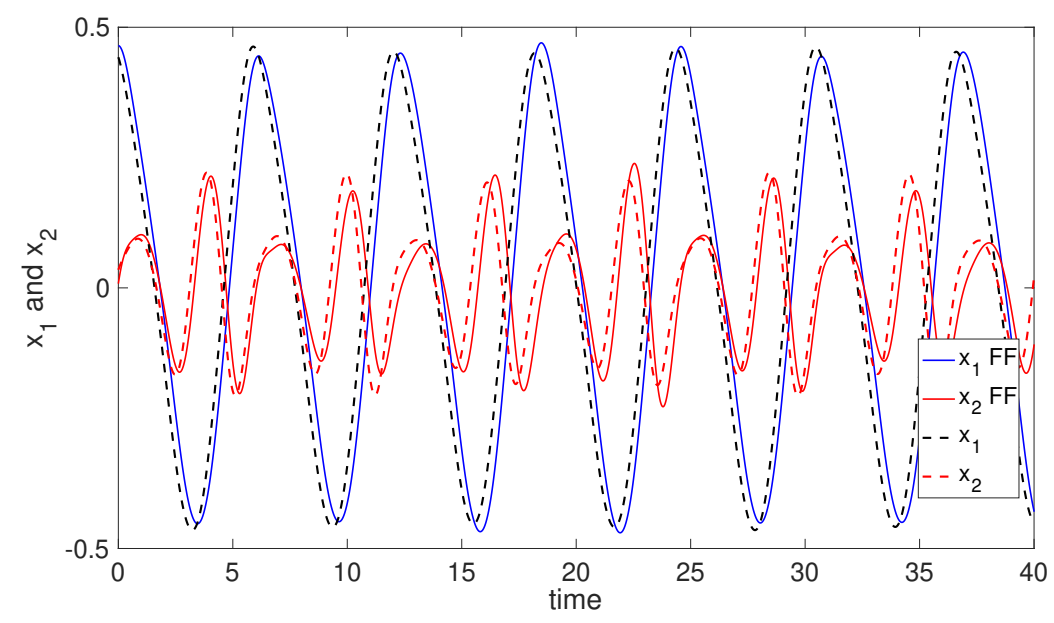

Figure 5: Comparison of the quasi-periodic solution computed using the Floquet forms (5) with the method described in section 2.4 and the quasi-periodic solution using the quasiperiodic HBM from [GVVC17]. The shapes qualitatively coincide very well.

\subsection{Forced systems}

We consider here some periodically forced (non-autonomous) dynamical systems that also encounter typical bifurcations such as saddle-node, pitchfork, symmetry breaking, period doubling and Neimark-Sacker bifurcations associated to changes of stability. In this case, all the $N$ Floquet multipliers $\rho_{n}, n=1, \ldots N$ are variables and analyzed to assess the stability of the periodic solutions.

\subsubsection{Forced Duffing oscillator}

Let us consider a classical Duffing oscillator

$$
\ddot{u}+\mu \dot{u}+\omega_{0}^{2} u+\Gamma u^{3}=F \cos (\Omega t)
$$

where $u(t)$ is the physical unknown we are interested in and $\lambda=\Omega$, the excitation frequency, is the control parameter we choose for computing the bifurcation diagram. Introducing $v=\dot{u}$ and $r=u^{2}$ yields the following quadratic system:

$$
\begin{array}{ll}
0=v & -\dot{u} \\
0=F \cos (\Omega t)-\mu v-\omega_{0}^{2} u-\Gamma u r & -\dot{v} \\
0=r-u^{2} &
\end{array}
$$



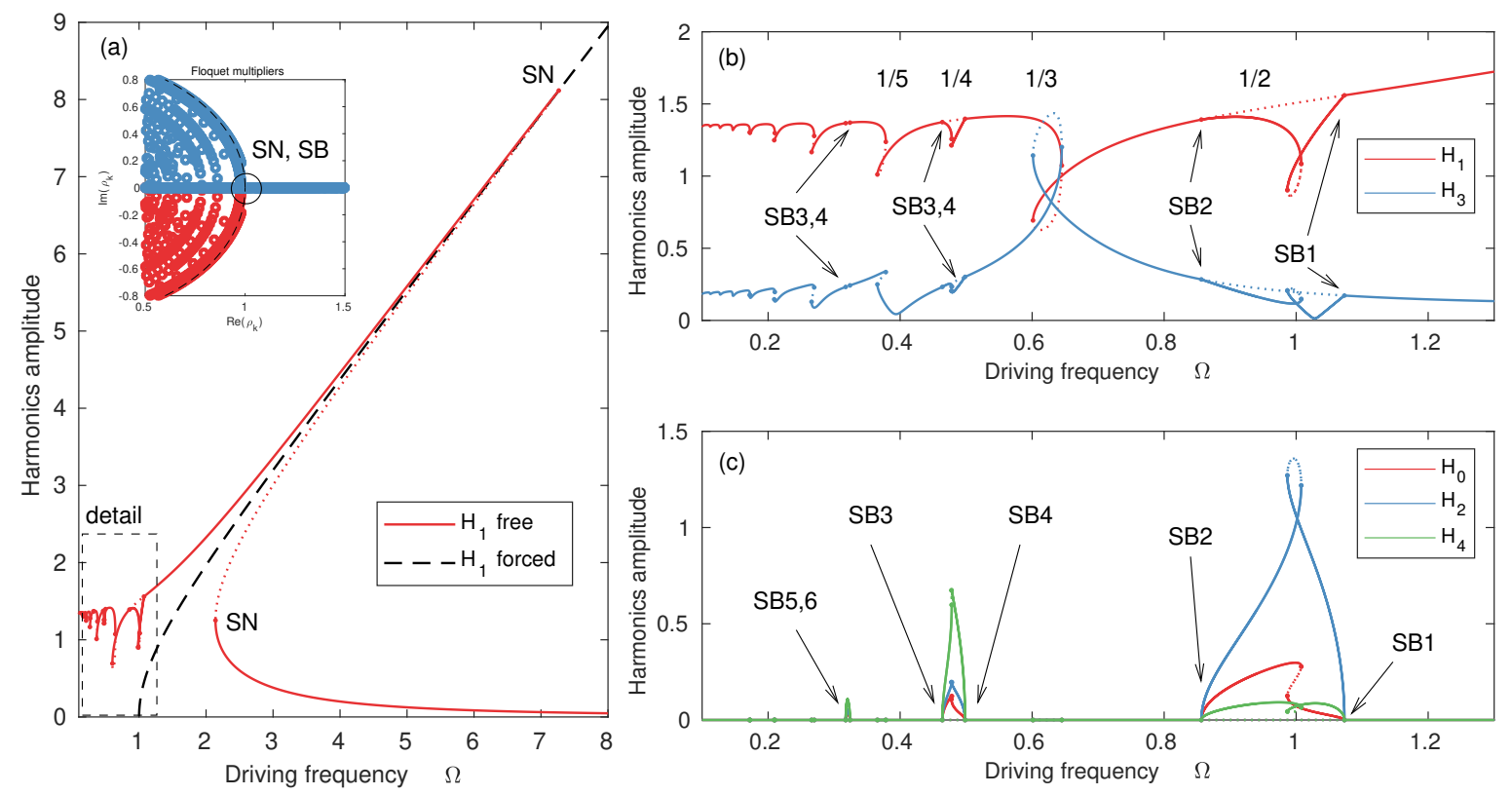

Figure 6: Bifurcation diagram for a Duffing oscillator. (a) Amplitude of the first harmonic of the periodic response $u(t)$ as a function of excitation frequency, for forced and free response, as mentioned. Full and dashed lines indicate stable and unstable branches, respectively. (b,c) Detail of the superharmonic resonances of order $1 / n, n \in\{2,3,4,5\}$. SB: symmetry breaking bifurcations; SN: saddle node bifurcations. (b) odd harmonics $H_{1}$, $\left.H_{3}\right)$; (c) even harmonics $H_{0}, H_{2}, H_{4}$ as mentioned. Parameters: $\mu=0.05, F=3, \omega_{0}=1$, $\Gamma=1, H=30$.

that can be implemented in MANLAB to compute the periodic solution $u(t)$ as well as its stability as a function of $\Omega$.

We first consider a continuation at fixed driving amplitude $F$ with the driving frequency $\Omega$ as the continuation parameter. The bifurcation diagram of $u(t)$ as a function of $\Omega$ is given in Figure 6 for $H=30, F=3, \mu=0.05, \omega_{0}=1, \Gamma=1$. This diagram is classical and can be found for example in [LT10] for numerical computation or in [NM79] for multiple scale analysis. The global bifurcation diagram showing the amplitude of the first harmonic of the periodic solution $u(t)$ is displayed in Figure 6(a). The shape of the branches qualitatively conforms to the one obtained by perturbation methods and the saddle node (SN) bifurcations are precisely located at the turning point. This latter remark highlights the fact that the Floquet exponents computed and sorted from the Hill matrix of $(21)$ crosses the imaginary axis at the correct $\Omega$. Figure 6(b) and (c) show a zoom for $\Omega \in[0.1,1.3]$ to highlight the behaviour of the system under superharmonic resonances, for which numerous SN bifurcations are obtained, again precisely located at the turning points. The superharmonic resonances of order $1 / n$ with $n$ odd are characterized by small resonances of the haramonics $H_{n}$, giving rise to loops of the resonance curves, as shown in Fig. 6(b). For the superharmonic resonances of order $1 / n$ with $n$ even, the branch of symmetric $u(t)$ (all even harmonics are zero) becomes unstable between two pitchfork bifurcations and gives birth to stable branches where $u(t)$ is asymmetric (non-zero even harmonics). Those pitchfork bifurcations, called consequently symmetry breaking (SB) bifurcation, are well recovered. For the chosen level of forcing $(F=3)$, even superhamonic resonances of order $1 / 2,1 / 4$ and $1 / 6$ are obtained. Those two types of bifurcations (SB, $\mathrm{SN}$ ) are responsible of the same behaviour of the Floquet multipliers, one of them crossing the unit-circle for both cases (see inset of Fig. 6(a)).

One has also to mention that the location of the SB bifurcation is exactly found where 
the bifurcation detector implemented in Manlab (based on the emergence of a power series in the Taylor series in the vicinity of a bifurcation point, see [CM13] for details) detects a branch crossing. This further highlights the quality of the stability detection of the proposed algorithm.
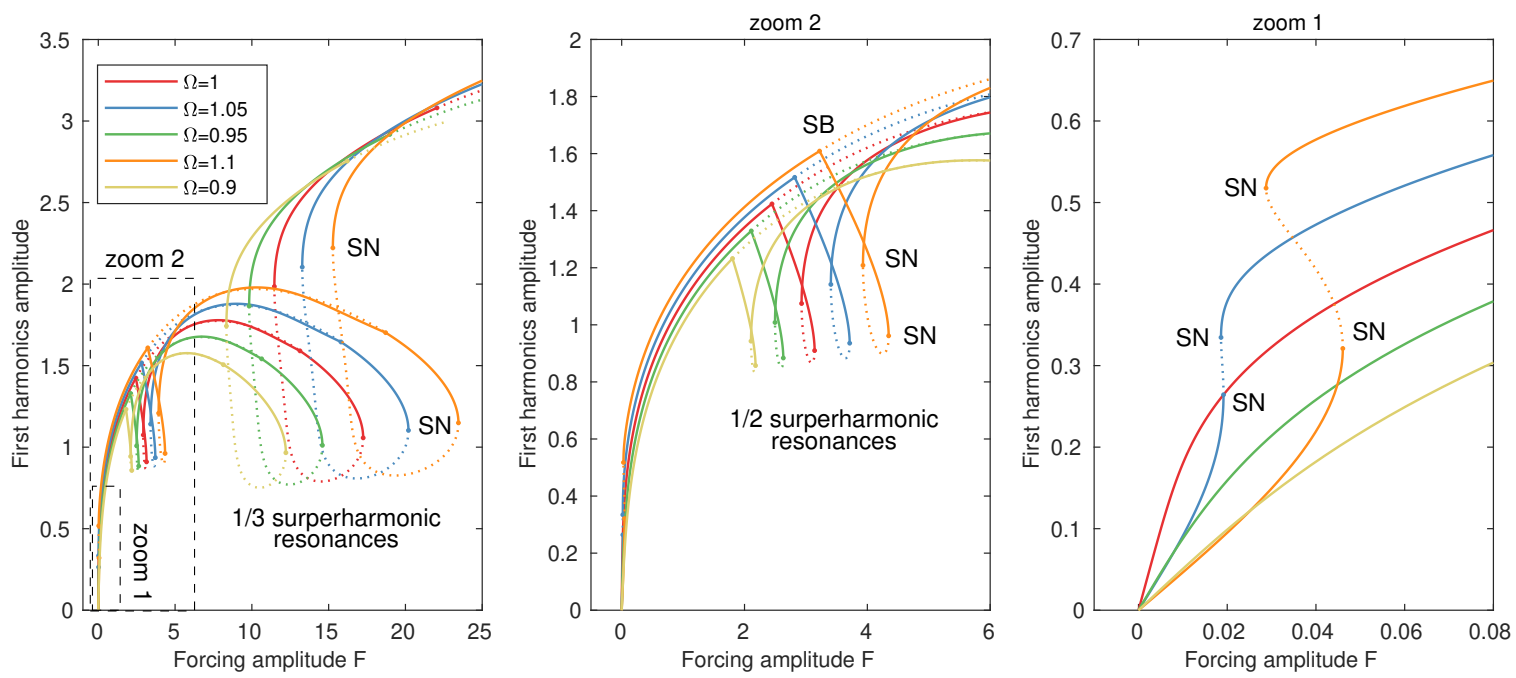

Figure 7: Bifurcation diagram for a Duffing oscillator with fixed driving frequency $\Omega$ $(\Omega \in\{0.9 ; 0.95 ; 1 ; 1.05 ; 1.1\}$, see the legend for the color of the curves) and the driving excitation $F$ as the bifurcation parameter. (a) whole diagram and (b,c) zoom on details. Only the first harmonic of $u(t)$ is plotted. Parameters: $\mu=0.05, \omega_{0}=1, \Gamma=1, H=30$.

We now consider a continuation at fixed driving frequency $\Omega$ with the driving amplitude $F$ as the continuation parameter. The bifurcation diagram of $u(t)$ as a function of $F$ is given in Figure 7 for $H=30, F=3, \mu=0.05, \omega_{0}=1, \Gamma=1$. For low values of $F$ (see Fig. 7(c)), the diagram is classical and can be found for example in [NM79]. In this case, since $\Gamma>0$, the behaviour is hardening and the two saddele-node bifurcations (SN) are obtained only for driving frequencies $\Omega$ higher that the natural frequency $\omega_{0}$. Then, for higher values of $F$ (see Figs. 7(a,b)), loops of superharmonic resonances of order $1 / 2$ and $1 / 3$ are observed, for any value of $\Omega$, showing symmetry breaking (SB) and saddle node (SN) bifurcation, as in the case of a continuation in $\Omega$ in Fig. 6.

\subsubsection{Parametric pendulum}

Parametric pendulums are archetypal examples of an important class of dynamical systems known as time-varying systems or parametric oscillators [Ric12, BL18, Laz19]. We consider the classical case of a pendulum forced by a vertical acceleration of its pivot that has notably been analytically treated in [BM90]. In the latter, the equation of motion is written in the form

$$
\ddot{\theta}+2 \delta \dot{\theta}+(1-\varepsilon \sin (\omega t)) \sin (\theta)=0
$$

where $\theta$ is the angular displacement from the downward vertical, $\delta$ is the damping ratio, $\varepsilon$ represents the forcing amplitude, $\omega$ is the ratio of the forcing frequency $\Omega$ to the natural frequency $\omega_{0}$ and the unit of time $t$ is the inverse of the natural frequency. Introducing $\psi=\dot{\theta}, F=\varepsilon \sin (\omega t), s=\sin (\theta), c=\cos (\theta)$ and the differentiated forms $\mathrm{d} s=c \mathrm{~d} \theta$, $\mathrm{d} c=-s \mathrm{~d} \theta$ (see [GCV19, GVC18] for more details on this recast) yields the following 
quadratic formulation :

$$
\begin{aligned}
& 0=\psi \quad-\dot{\theta} \\
& 0=-2 \delta \psi-(1-F) s-\dot{\psi} \\
& 0=F-\varepsilon \sin (\omega t) \\
& 0=s-\sin (\theta) \quad d s-c d \theta=0 \\
& 0=c-\cos (\theta) \quad d c+s d \theta=0
\end{aligned}
$$

To qualitatively compare the computed numerical results to the analytical solutions plotted in [BM90], we choose the parameters $\delta=1 / 8, \varepsilon=1.05$ and a truncation number $H=100$ to ensure convergence of the numerical Fourier series expansion of $\theta(t)$. The bifurcation parameter is chosen as the driving frequency so that $\lambda=\omega$.
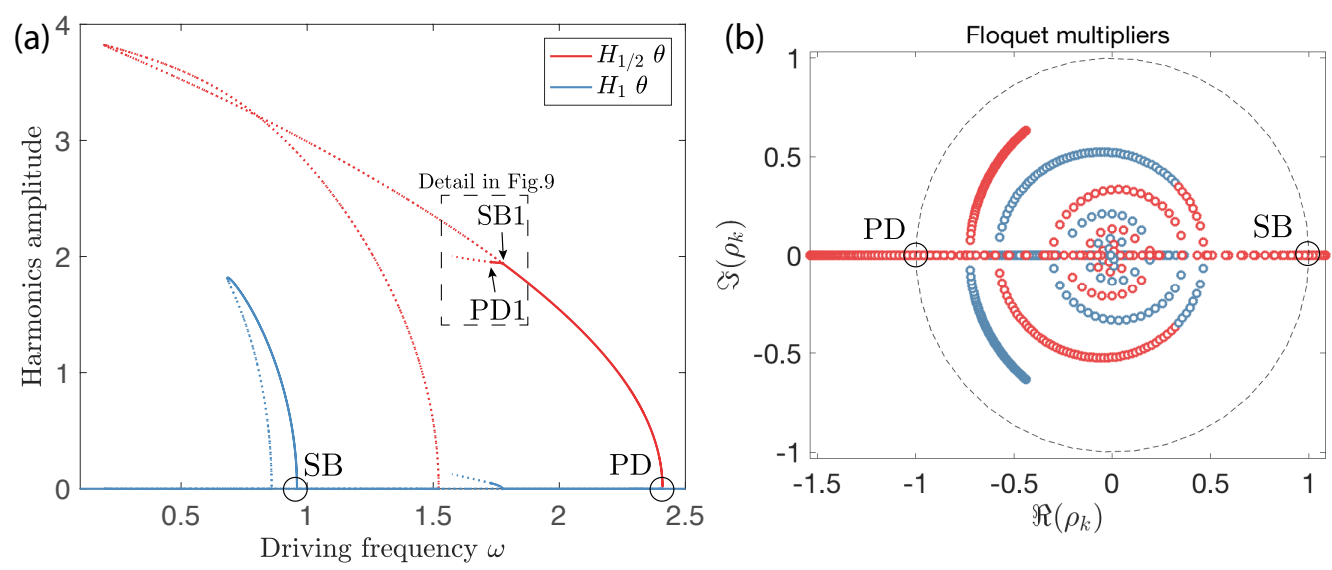

Figure 8: Bifurcation and local stability analysis of the nonlinear parametric pendulum of [BM90]. (a) First and second parametric resonances showing the amplitudes of harmonics $1 / 2$ and 1 of $\theta(t)$ as a function of $\omega$. (b) Locus of the Floquet multipliers in the complex plane for the trivial branch $\theta(t)=0$ when $\omega$ varies from 0.2 to 2.8 .

Figures 8 and 9 show a numerical evolution of $\theta(t)$ and a stability prediction as expected from the analytical study of [BM90]. As shown with the Floquet multipliers in Figure
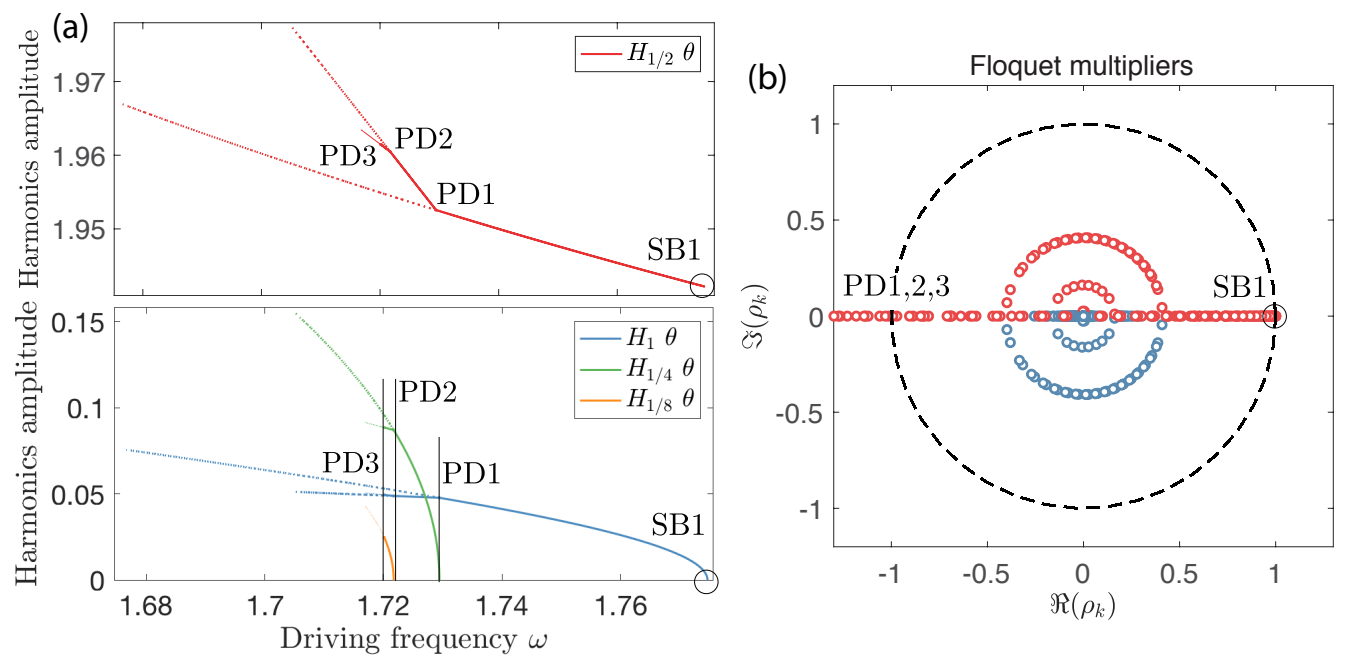

Figure 9: Bifurcation and local stability analysis of the beginning of the period-doubling cascade. (a) Zoom on the period doubling sequence showing the amplitudes of harmonics $1 / 8,1 / 4,1 / 2$ and 1 of $\theta(t)$ as a function of $\omega$. (b) Locus of the Floquet multipliers associated with the bifurcation diagram in (a). 
8(b) when $\omega$ varies, the trivial downward vertical solution $\theta(t)=0$ alternates between $T$-periodic and $2 T$-periodic instability regions (with $T=2 \pi / \omega$ ) that respectively give rise to Simple (SB) and Period Doubling (PD) bifurcations. The amount of damping in our example, $\delta=1 / 8$, limits the number of instability regions to two. For $\omega \approx 1\left(\Omega \approx \omega_{0}\right)$, the parametric forcing destabilizes the trivial downward equilibrium position $\theta(t)=0$ which bifurcates on an asymmetric oscillation dominated by the lowest harmonic $\omega$. The principal parametric instability region of the trivial solution $\theta(t)=0$ is located around $\omega=2\left(\Omega \approx 2 \omega_{0}\right)$. The sequence of bifurcation emerging from this $2 T$-periodic instability is complex and will be described from right to left as a function of $\omega$. At $\omega \approx 2.4$, the parametric forcing destabilizes the trivial downward equilibrium position $\theta(t)=0$ on a twice slower symmetric motion with fundamental frequency $\omega / 2$. Continuing on the new stable branch, around $\omega=1.8$, a symmetry breaking bifurcation occurs and harmonics multiple integers of $\omega$ emerge in the solution, as shown by the curve $H_{1} \theta$ in Figure 8(a). Decreasing $\omega$ even further, a new period doubling bifurcation occurs which is the beginning of a period-doubling cascade that is detailed in Figure 9.

In order to follow the period doubling bifurcations of Figure 8, one needs to add harmonics of order $1 / n$, where $n$ is a positive integer, in the Fourier expansion of the Harmonic Balance Method each time a period doubling bifurcation is encountered. In practice, this is done by forcing the oscillator at $n \omega$, and by computing a response with a fundamental harmonic lower than $n$. Thus, the red branch with harmonics $1 / 2$ shown in Figures 8-9 has been obtained by forcing the oscillator at a $2 \omega$ frequency and by computing a response with fundamental harmonic $\omega$. Around $\omega=1.73$ in Figure 9(a), a new period doubling bifurcation (PD1) occurs as shown by the associated Floquet multipliers crossing the unit circle on the real axis by the left. To compute the new emerging solution with harmonics $1 / 4$, we had to force the oscillator at a $4 \omega$ frequency to be able to represent a solution with a full spectrum with fundamental harmonic $\omega$. Decreasing $\omega$ further, the stable branch encounters a new period doubling bifurcation PD2 where a new solution with harmonic $1 / 8$ arises. Although we stopped computing this sequence after PD3, it is well known that the period-doubling sequence actually terminates with a narrow band of nearly-periodic oscillations before chaotic oscillations or some independent periodic oscillations are reached [BM90]. The period-doubling cascade is correctly computed from our stability algorithm. This cascade is also recognizable with Floquet multipliers in the Argand plane that consist of sequence of circles of conjugate eigenvalues, with smaller and smaller radius, that lock-in on the real axis so that Floquet multipliers cross the unit circle from the left.

\subsubsection{Coupled oscillators in 1:2 internal resonance}

Let us consider the following dynamical system introducing two oscillators in one-to-two (1:2) internal resonance:

$$
\left\{\begin{array}{l}
\ddot{u}_{1}+\mu_{1} \dot{u}_{1}+\omega_{1}^{2} u_{1}+\beta_{1} u_{1} u_{2}=F_{1} \cos \Omega t \\
\ddot{u}_{2}+\mu_{2} \dot{u}_{2}+\omega_{2}^{2} u_{2}+\beta_{2} u_{1}^{2}=F_{2} \cos \Omega t
\end{array}\right.
$$

where the two natural frequencies are chosen such that $\omega_{2} \simeq 2 \omega_{1}$ and where only the corresponding resonant quadratic nonlinear terms have been kept in the oscillators [GH83a, TTC04]. Since the above system is naturally quadratic, one has just to introduce $v_{1}=\dot{u}_{1}$ and $v_{2}=\dot{u}_{2}$ to obtain a first order system in the right formalism without auxiliary equations $\left(\boldsymbol{x}_{a}=\boldsymbol{g}_{a}=\mathbf{0}\right.$ in Eqs. (6)). This kind of system is encountered in many different physical systems and has already been studied by a number of investigators (see e.g. [NM79, NB89, Nay00] and references therein, about spring pendulums, ships, surface waves etc.). It is also the canonical system for studying more complex quadratic internal resonances such as 1:1:2, 1:2:4 etc. encountered in shells and musical instruments [TTC05, TTL07, MTTB14, 
MTT15]. Here, as previously, the bifurcation parameter is chosen as the driving frequency $\lambda=\Omega$.

Two main canonical cases are considered here. The first one is when only the low frequency oscillator is forced, namely $F_{2}=0$. In this case, a coupled solution between the two oscillators is obtained, for which, at first order, $u_{1}$ oscillates at frequency $\Omega$, $u_{1}(t) \simeq a_{1} \cos \left(\Omega t+\varphi_{1}\right)$ and $u_{2}$ oscillates at frequency $2 \omega, u_{2} \simeq a_{2} \cos \left(2 \Omega t+\varphi_{2}\right)$, where $a_{1}$, $a_{2}$ are the amplitudes and $\varphi_{1}, \varphi_{2}$ are the phases. Fig. 10(a) shows $a_{1}$ and $a_{2}$ as a function of $\Omega$. Several saddle node (SN) bifurcations are obtained and successfully computed at the limit points of branches (where the tangent is vertical). Moreover, a zone of quasi-periodic solution is predicted around $\omega=\omega_{1}$, obtained after Neimark-Sacker (NS) bifurcations, for which two complex conjugate Floquet multipliers exit the unit circle (Fig. 10(b)). This case if fully compatible with approximate analytical solutions [NM79].

The second considered case is when only the high frequency oscillator is forced, namely $F_{1}=0$. In this case, an uncoupled solution, for which the unforced mode has no oscillations $\left(u_{1}(t) \equiv 0, u_{2}(t)=a_{2} \cos \left(\Omega t+\varphi_{2}\right)\right)$ coexists with a coupled solution for which the low frequency mode oscillates at half the driving frequency $\left(u_{1}(t)=a_{1} \cos \left(\Omega / 2 t+\varphi_{1}\right), u_{2}(t)=\right.$ $\left.a_{2} \cos \left(\Omega t+\varphi_{2}\right)\right)$. Fig. 11(a) shows $a_{1}$ and $a_{2}$ as a function of $\Omega$. The branches of two solutions are connected by period doubling (PD) bifurcations, for which a real Floquet multiplier exits the unit circle by the left. In practice, if the period 1 solution is computed with a standard HBM method, the stability check detects the PD bifurcations, as shown in Fig. 11(b). Then, to compute the coupled period 2 solutions, one has to add harmonics of order $1 / 2$ in the Fourier expansions of the HBM. This is done in practice by forcing the second oscillator at a $2 \Omega$ frequency. The uncoupled solution has thus zero odd harmonics and the PD bifurcations degenerate into pitchfork bifurcations, from which the coupled solutions emerge with a full spectrum, its fundamental harmonics being the harmonics of order $1 / 2$ of the classical coupled solution.
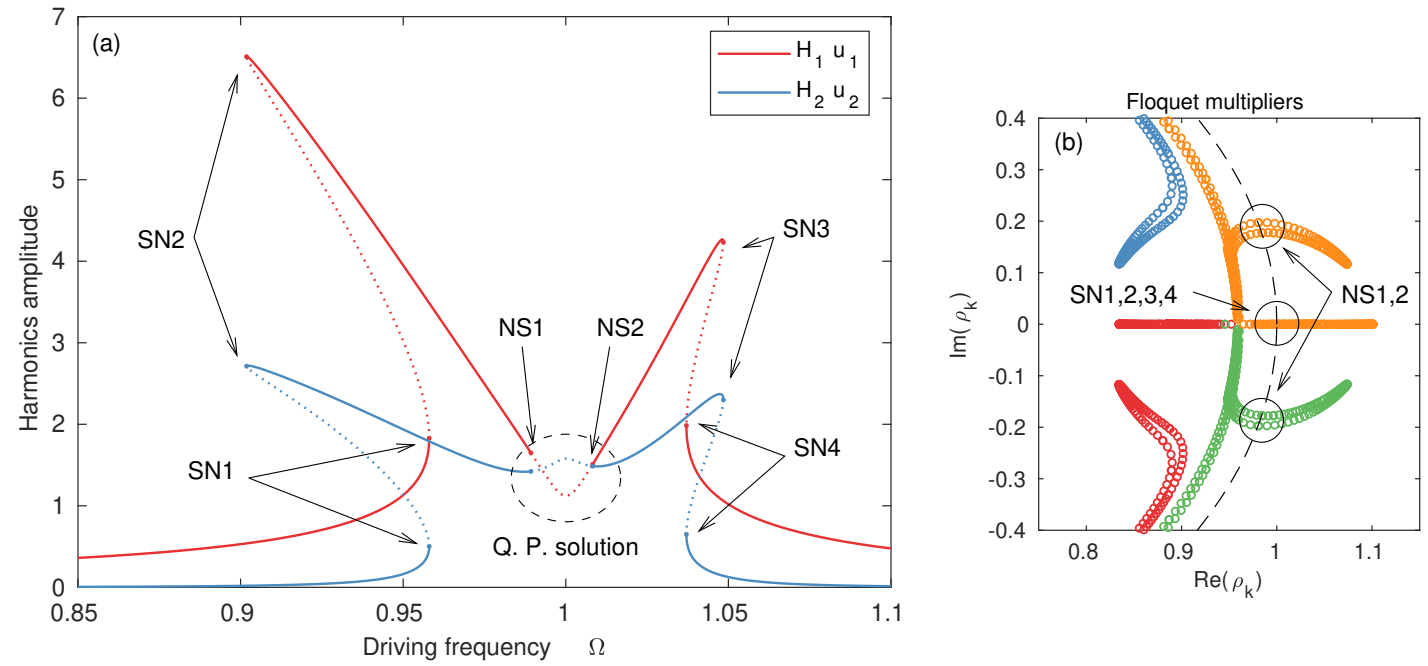

Figure 10: Bifurcation diagram for two oscillators in 1:2 internal resonance. (a) Amplitude of harmonics 1 of $u_{1}$ and harmonics 2 of $u_{2}$; (b) locus of the Floquet multipliers in the complex plane. Forcing of the low frequency oscillator $\left(F_{2}=0\right)$ around its resonance $\left(\Omega \simeq \omega_{1}\right)$. Value of the parameters: $\omega_{1}=1 ; \omega_{2}=2 ; \mu_{1}=0.01 ; \mu_{2}=0.02 ; \beta_{1}=\beta_{2}=0.1$; $F_{1}=0.1 . H=10$ harmonics have been retained in the computation. 

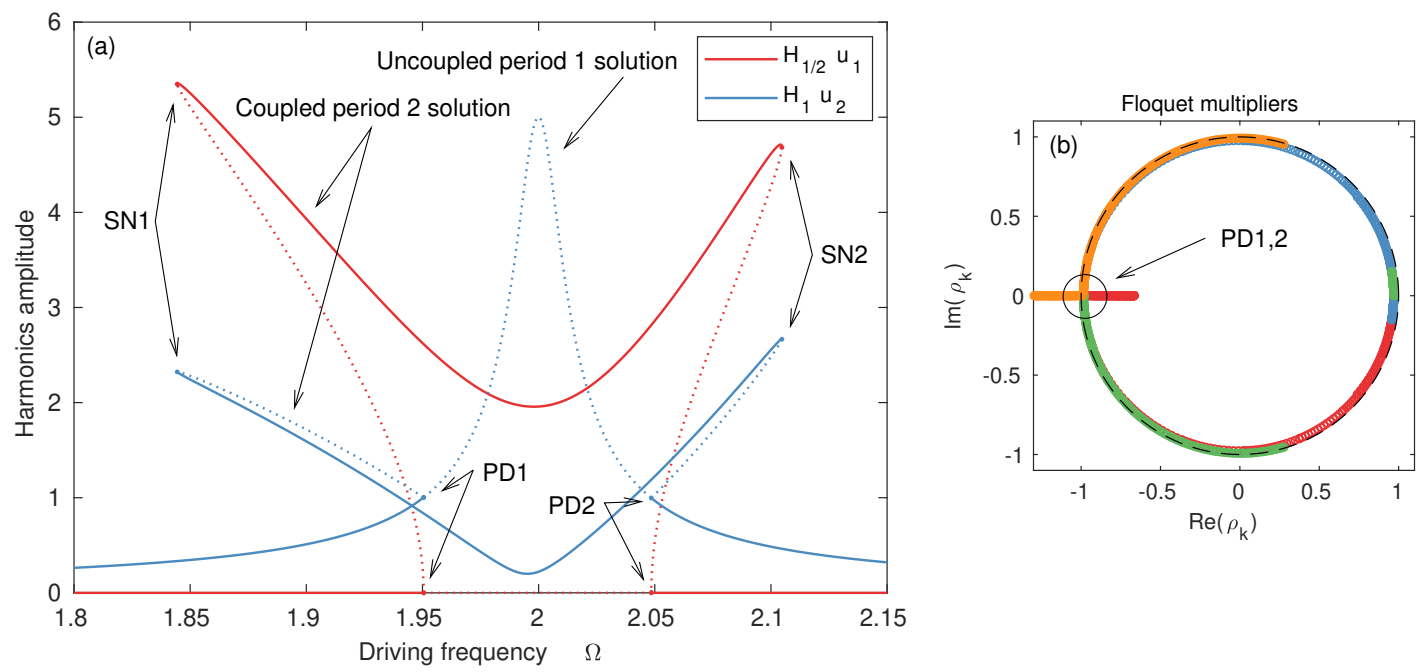

Figure 11: Bifurcation diagram for two oscillators in 1:2 internal resonance. (a) Amplitude of harmonics $1 / 2$ of $u_{1}$ and harmonics 1 of $u_{2}$; (b) locus of the Floquet multipliers in the complex plane for the uncoupled branch. Forcing of the high frequency oscillator $\left(F_{1}=0\right)$ around its resonance $\left(\Omega \simeq \omega_{2}\right)$. Value of the parameters: $\omega_{1}=1 ; \omega_{2}=2 ; \mu_{1}=0.01$; $\mu_{2}=0.02 ; \beta_{1}=\beta_{2}=0.1 ; F_{2}=0.2 . \quad H=10$ harmonics have been retained in the computation.

\subsection{Autonomous conservative systems}

The case of autonomous conservative system is particular, as compared to generic autonomous (dissipative) systems considered in section 3.2, since they have an invariant quantity (a first integral). In our case, it is the total mechanical energy $\mathcal{H}$ of our systems. Ideed, the system periodic orbits belong to a one-parameter family, parametrized by the value of $\mathcal{H}$ which should be the bifurcation parameter and which do not appear explicitely in the equations of the dynamical system $\left[\mathrm{MAFG}^{+} 03\right]$. This fact has two consequences in our context of resonant mechanical systems.

Firstly, to ensure the uniqueness of the computed periodic orbit with an appropriate phase condition, one has to add a fictitious bifurcation parameter $\lambda$ in the dynamical system by adding a term $\lambda \dot{u}$ (all details can be found in $\left[\mathrm{MAFG}^{+} 03, \mathrm{KCV} 13, \mathrm{KCV} 14\right]$ ). In our case the phase condition is $\dot{u}_{1}(0)=0$, i.e the derivative of the first state variable vanishes at $t=0$.

Secondly, the autonomous conservative systems considered here are associated to the free oscillations of resonant mechanical systems and lead to the definition of nonlinear modes of conservative systems [SP93, TTC04, KPGV09, RKC16]. Usually, the frequency $\omega$ of those modes is displayed as a function of $\mathcal{H}$, leading to so-called frequency-energy plots [KPGV09]. One can also choose a particular coordinate of the system and plot its amplitude as a function of $\omega$, leading to so-called backbone curves [NM79]. Both representations will be used in the following.

About the stability of those periodic solutions, since the systems considered here are autonomous, as explained in section 3.2, one of the Floquet multipliers is always $\rho_{1}=1$ and is not considered in the stability checks. Moreover, since the dynamical system is conservative, a classical case is to obtain periodic solutions which are marginally stable. In this case the tolerance for the stability check has to be chosen carefully as the multipliers often lies exactly on the unit cycle. Numerically it is then irrelevant to ask for $\rho_{n} \leq 1$ and a numerical threshold $\nu$ must be added so that the stability condition becomes $\rho_{n} \leq 1+\nu$. 
In practice, $\nu=10^{-6}$ is used.

\subsubsection{Duffing oscillator}

The equation of motion of the free Duffing oscillator corresponding to the forced one presented in (33) is

$$
\ddot{u}+\lambda \dot{u}+\omega_{0}^{2} u+\Gamma u^{3}=0,
$$

where, as explained above, a dissipative term $\lambda \dot{u}$ has been added, where $\lambda$ is a free (bifurcation) parameter. Since this term is dissipative, $\lambda$ is found very close to zero, as a result of the computation of periodic orbits, that exist theoretically only if the system is conservative $(\lambda=0)$. The phase condition is $\dot{u}(0)=0$.

The bifurcation diagram of the system is superimposed with the bifurcation diagram 6(a) of the forced Duffing oscillator, constituting its backbone curve. The whole branch is found marginally stable, with the second Floquet exponents $\rho_{2}$ computed close to one with a $10^{-13}$ accuracy. The bifurcation parameter $\lambda$ is found equal to zero at a numerical error less than $10^{-14}$. Those result confirm the efficiency of the proposed algorithm.

\subsubsection{A two degrees of freedom mass spring nonlinear system}
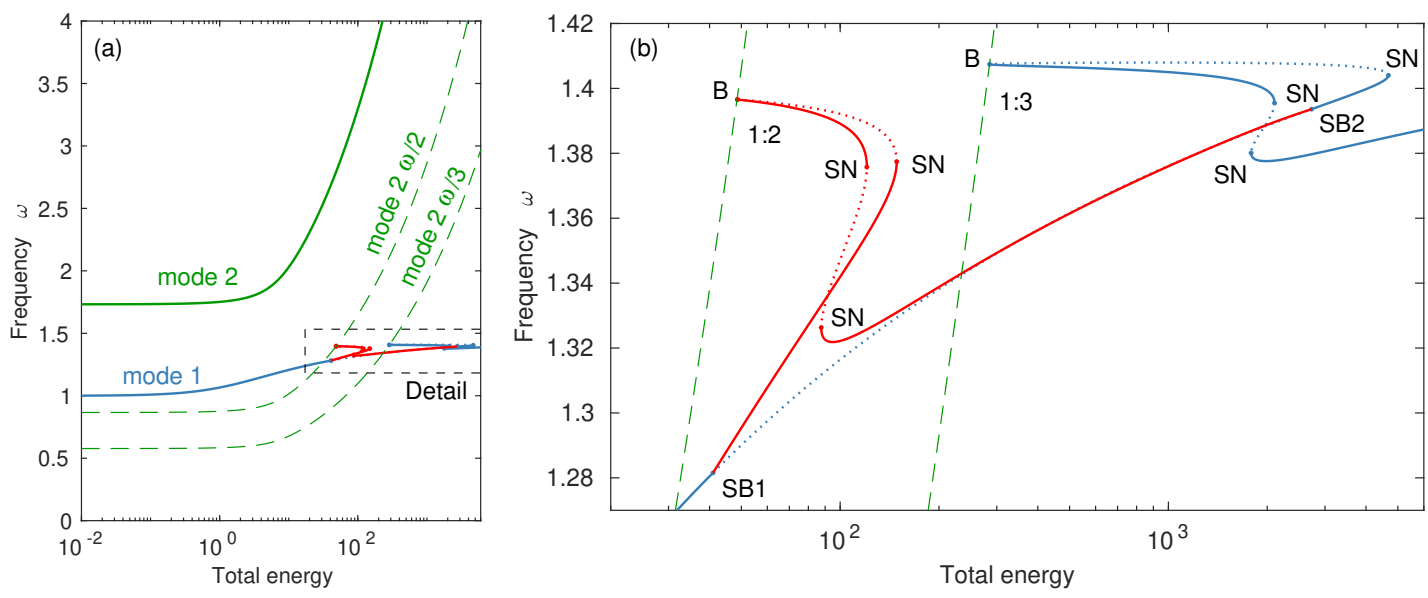

Figure 12: (a) The two nonlinear modes of the two degrees of freedom mass spring system with a cubic nonlinear spring. Frequency $\omega$ of periodic orbits as a function of the total energy $\mathcal{H}$ of the system. Logarithmic scales. (b) detail of (a); '--': branches of mode 2 with frequency divided by 2 or 3 , that are connected to the tongues of the 1:2 and 1:3 internal resonances. $H=30$ harmonics have been retained in the computation.

We consider here a two degrees of freedom system composed of two masses (of inertia 1) linked to each other and to the frame by three linear springs (of equal stiffness 1 ). A nonlinear spring with a cubic restoring force also connects one mass to the frame. The equations of motion are:

$$
\left\{\begin{array}{l}
\ddot{u}_{1}+\lambda \dot{u}_{1}+2 u_{1}-u_{2}+u_{1}^{3} / 2=0 \\
\ddot{u}_{2}+\lambda \dot{u}_{2}-u_{1}+2 u_{2}=0
\end{array}\right.
$$

where, as explained above, dissipative terms $\lambda \dot{u}_{1}, \lambda \dot{u}_{2}$ have been added. This system has been used in several papers about nonlinear modes (see [KPGV09, RKC16] for instance). Its total energy is:

$$
\mathcal{H}=1 / 2\left[\dot{u}_{1}^{2}+\dot{u}_{2}^{2}+u_{1}^{2}+u_{2}^{2}+\left(u_{1}-u_{2}\right)^{2}+u_{1}^{4} / 4\right] .
$$


Introducing $v_{1}=\dot{u}_{1}, v_{2}=\dot{u}_{1} r=u_{1}^{2}$ yields the following quadratic system:

$$
\begin{array}{ll}
0=v_{1} & -\dot{u}_{1} \\
0=v_{2} & -\dot{u}_{2} \\
0=-\lambda v_{1}-2 u_{1}+u_{2}+u_{1} r / 2 & -\dot{v}_{1} \\
0=-\lambda v_{2}+u_{1}-2 u_{2}+ & -\dot{v}_{2} \\
0=r-u_{1}^{2} &
\end{array}
$$

In practice, since we need $\mathcal{H}$ to compute the frequency energy plots, it is possible to add Eq. (40) (which is naturally quadratic with $u_{1}^{4}=r^{2}$ ) in (41) to compute it as an additional auxiliary variable. This slightly reduces the accuracy of the computations, since the polynomial order of variables in $\mathcal{H}$ is higher than in the remaining part of the system which increases the number of Fourier coefficients needed to obtain the same accuracy in the HBM method. Then, one consider only the static part $\left(H_{0}\right)$ of $\mathcal{H}$ for the plots, naturally given by the HBM as the zero-th. harmonic of the Fourier expansion of $\mathcal{H}$.

The two degree of freedom system (39) has two nonlinear modes that can be defined as two families of periodic orbits that emerge from its two natural frequencies $\omega_{1}=1$ and $\omega_{2}=\sqrt{3}$ [RKC16]. Fig. 12 shows the frequency $\omega$ of the periodic orbits associated to the two nonlinear modes as a function of the energy $\mathcal{H}$ of the system. Mode 2 is stable along its whole branch, whereas mode 1 in subjected to internal resonances $1: n, n=2,3,4 \ldots$ with mode 2 , that create tongues in the frequency energy plot (FEP). For odd internal resonance ( $n$ odd), the tongue emerges from the main branch without pitchfork bifurcations and is only subjected to saddle node (SN) bifurcations. For even internal resonance ( $n$ even), the main branch is unstable in the vicinity of the tongue, the latter being connected to the main branch with two symmetry breaking (SB) bifurcations. It also shows SN bifurcations. All those bifurcations are accurately predicted by the stability check [KPGV09]. In particular, all SN bifurcation are detected at the limit point of vertical tangent in the FEP (the energy is the bifurcation parameter) and SB bifurcations occur at positions predicted by the branch point detector of [CM13].

Another feature is that each 1:n internal resonance tongue is connected in a particular point (denoted by B in Fig. 12) to the branch of mode 2 with its frequency $\omega$ divided by $n$. At this point, there is a change of stability on the tongue branch, which is accurately predicted by the stability detector. This point is also particular since three branches of solution of the HBM algebraic system (14) intersect in this point (the branch of mode 2 and two branches for mode 1 : if $\left(u_{1}, u_{2}\right)$ is solution, $\left(-u_{1},-u_{2}\right)$ is also solution with the same energy $\mathcal{H})$.

\subsection{A hinged-hinged beam}

This section presents the case of a hinged-hinged beam, studied in particular in [Lew97] for the free vibration (autonomous conservative) case and in [Lew97, LTD12] for the forced and damped case. This is an example of a system of a larger size in terms of numbers of degrees of freedom than the previous ones, selected to demonstrate the performances of the proposed method.

\subsubsection{Governing equations}

We consider a straight beam of length $L$, with a homogeneous cross section of area $S$ and second moment of area $I$ built in an homogeneous and isotropic elastic material of Young's modulus $E$ and density $\rho$. Its large amplitude vibrations are modelled here by the following von Kármán model with neglected axial inertia and immovable ends in the axial direction 
[WK50, Lew94b]:

$$
\left\{\begin{array}{l}
\rho S w_{, t t}+E I w_{, x x x x}-N w_{, x x}=p, \\
N=\frac{E S}{2 L} \int_{0}^{L} w_{, x}^{2} \mathrm{~d} x
\end{array}\right.
$$

where $w(x, t)$ is the transverse displacement at axial position $x$ and time $t, N(t)$ is the axial force, that is uniform over the length beam, $p(x, t)$ is the external force per unit length and $\bullet_{, x}=\partial \bullet / \partial x, \bullet, t=\partial \bullet / \partial t$. For numerical purpose and also to reduce the number of free parameters, this model is rewritten in the following dimensionless form:

$$
\left\{\begin{array}{l}
\ddot{\bar{w}}+\bar{w}^{\prime \prime \prime \prime}-\bar{N} \bar{w}^{\prime \prime}=\bar{p} \\
\bar{N}=\frac{1}{2} \int_{0}^{1} \bar{w}^{\prime 2} \mathrm{~d} \bar{x}
\end{array}\right.
$$

with:

$$
\bar{w}=\frac{w}{r}, \quad \bar{x}=\frac{x}{L}, \quad r=\sqrt{\frac{I}{S}}, \quad \bar{t}=\frac{1}{L^{2}} \sqrt{\frac{E I}{\rho S}} t, \quad \bar{N}=\frac{L^{2}}{E I} N, \quad \bar{p}=\frac{S L^{4}}{E I^{2}} p .
$$

$r$ is the radius of gyration of the beam, $\bullet^{\prime}=\partial \bullet / \partial \bar{x}$ and $\bullet=\partial \bullet / \partial \bar{t}$.

The model of Eqs. (43a,b) is expanded on $K$ eigenmodes of its linear part (of natural frequency $\omega_{k}$ and mode shape $\Phi_{k}(\bar{x}), k=1 \ldots K$ :

$$
\bar{w}(\bar{x}, \bar{t})=\sum_{k=1}^{K} \Phi_{k}(\bar{x}) q_{k}(\bar{t}) .
$$

Using the orthogonality properties, it can be shown that the modal coordinates $q_{k}(t)$ verify the following dynamical system:

$$
\left\{\begin{array}{l}
\ddot{q}_{k}+2 \xi_{k} \omega_{k} \dot{q}_{k}+\omega_{k}^{2} q_{k}+\bar{N} \sum_{i=1}^{K} \alpha_{i k} q_{i}=Q_{k}, \\
\bar{N}=\frac{1}{2} \sum_{i, j=1}^{K} \alpha_{i j} q_{i} q_{j},
\end{array}\right.
$$

where

$$
\alpha_{i j}=\alpha_{j i}=-\int_{0}^{1} \Phi_{i}(\bar{x}) \Phi_{j}^{\prime \prime}(\bar{x}) \mathrm{d} \bar{x}=\int_{0}^{1} \Phi_{i}^{\prime}(\bar{x}) \Phi_{j}^{\prime}(\bar{x}) \mathrm{d} \bar{x}, \quad Q_{k}=\int_{0}^{1} \Phi_{k}(\bar{x}) \bar{p}(\bar{x}, t) \mathrm{d} \bar{x} .
$$

One can verify that the above first equation is valid for most classical boundary conditions. Otherwise, different coefficients for the nonlinear terms appear in Eqs. (46a,b).

For hinged-hinged boundary conditions, the transverse displacement verifies $w(0, t)=$ $w(L, t)=0, w^{\prime \prime}(0, t)=w^{\prime \prime}(L, t)=0$, the dimensionless natural frequencies $\omega_{k}=k^{2} \pi^{2}$, the mode shapes $\Phi_{k}(\bar{x})=\sqrt{2} \sin k \pi \bar{x}$ and the coefficients of the linear terms $\alpha_{k k}=k^{2} \pi^{2}$ and $\alpha_{i j}=0, \forall i \neq j$. The dynamical system (46) is then, for all $k=1, \ldots K$ :

$$
\left\{\begin{array}{l}
\ddot{q}_{k}+2 \xi_{k} \omega_{k} \dot{q}_{k}+\omega_{k}^{2} q_{k}+\pi^{2} k^{2} \bar{N} q_{k}=Q_{k}, \\
\bar{N}=\frac{\pi^{2}}{2} \sum_{i=1}^{K} i^{2} q_{i}^{2} .
\end{array}\right.
$$

In all the plots of this section (Figs. 13, 14, 15), the maximum amplitude of the dimensionless transverse displacement $\max _{t \in[0 T]} \bar{w}\left(x_{\text {disp }}, t\right)$ over one period $T=2 \pi / \Omega$, at a given position $x_{\text {disp }}$ on the beam, is shown. Its is reconstructed knowing the Fourier coefficients of $q_{k}(t)$ and the modal expansion (45). 


\subsubsection{Free response}
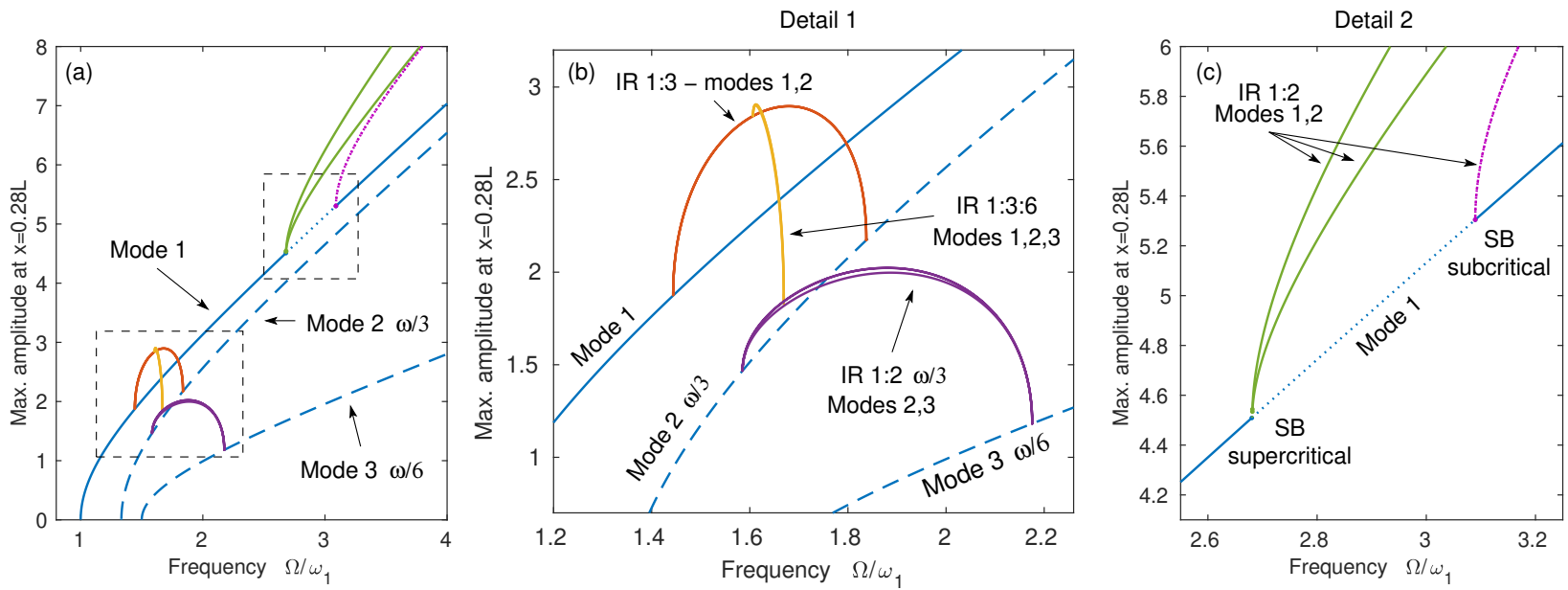

Figure 13: Backbone curves of a hinged-hinged beam. Maximum amplitude of the transverse displacement, over one period, at $x_{\text {disp }}=0.28 \mathrm{~L}$. (a) general view showing the backbone curve of the first mode and the multiple internal resonances originating from it; The backbones curves of mode 2 and 3, with frequency divided by 3 or 6 , are shown in '- -'; (b) detail of the 1:3 internal resonances between modes 1,2 and modes 2,3 and the 1:3:6 internal resonances between modes $1,2,3$; (c) 1:2 internal resonances between modes 1,2 with symmetry breaking (SB) bifurcations. $H=20$ harmonics and $K=10$ modes have been retained in the computations. All curves correspond to stable periodic solutions unless around the 1:2 internal resonance of the mode 1 backbone.

The free response of the hinged-hinged beam is obtained by looking for periodic solutions of (48) with the forcing and the damping terms cancelled $\left(Q_{k}=0, \xi_{k}=0\right.$ for all $k=1, \ldots K)$. An autonomous conservative system is obtained. As described in section 3.4, a term $\lambda \dot{q}_{k}$ is added in all equations (48a) to ensure the uniqueness of the periodic solution. In our computations, $H=20$ harmonics and $K=10$ modes have been chosen. The size of the dynamical system is here $K+1=11$ and the size of the Hill matrix, for the stability computation, is $(K+1)(2 H+1)=451$.

Firstly, the backbone curves of the first three modes of the beam are computed with their stability. In practice, for the $k$-th. mode, the simulation is initialized on the $k$-th. mode shape with a small amplitude $\left(q_{k}=0.001 \cos \omega_{k} t, q_{i}=0\right.$ for all $\left.i \neq k\right)$. The phase condition is prescribed on the $k$-th. modal coordinate: $\dot{q}_{k}(0)=0$. Those three backbones curves are shown in blue in Fig. 13. They are found marginally stable in a wide range of amplitude, with the modulus of all Floquet exponents smaller than $1+10^{-6}$. Mode 1 looses its stability around $\Omega=3 \omega_{1}$ where a 1:2 internal resonance with mode 2 is observed.

Those backbone curves are connected by internal resonance branches with a complex topology shown in Figs. 13(a,b). Around $\Omega=1.5 \omega_{1}$, a $1: 3$ internal resonance between modes 1 and 2 is observed. Two corresponding branches, of the same amplitude but with different phases, connect the two backbones (the one of mode 1 and the one of mode 2 with its frequency divided by 3). They have the shape of an arch and are shown in orange in Fig. 13(b). This 1:3 internal resonance is well documented in a series of article [Lew94a, Lew94b, Lew97] and emerges from the backbone of mode 1 without any loss of stability. The corresponding bifurcation is then not a simple pitchfork. Other internal resonances are also found: a 1:2 internal resonance between modes 2 and 3, that connects their backbones, shown in purple in Fig. 13(b) and a 1:3:6 internal resonance between modes 1,2 and 3 , shown in yellow. All the branches of those internal resonances are 
found marginally stable. Again, the branch points found at the connections between those curves are not simple pitchforks, since no loss of stability is observed. In practice, to find the branch point, a perturbation is added to the dynamical system and the continuation process naturally switch from the main branch to the bifurcated one [PF79]. One can also notice the very complex topology of those internal resonances, very different from the more classical loops (tongue) of the backbone curves often reported (see e.g. [KPGV09, GGTD19]) and shown in Fig. 12. To the knowledge of the author, this is the first time that such particular internal resonances are reported, especially the 1:3:6 one.

Another internal resonance is also found, that emerges from the backbone curve of mode 1 around $\Omega=3 \omega_{1}$. It is a 1:2 internal resonance with mode 2 , for which standard supercritical and subcritical pitchfork bifurcations are found, with a loss of stability of the main backbone in between. Two stable and two unstable branches emerge from the backbone of mode 1, that do not connect to each other and tends to infinity. Again, the classical internal resonance loop of [KPGV09], shown in Fig. 12, is not recovered.

\subsubsection{Forced response}
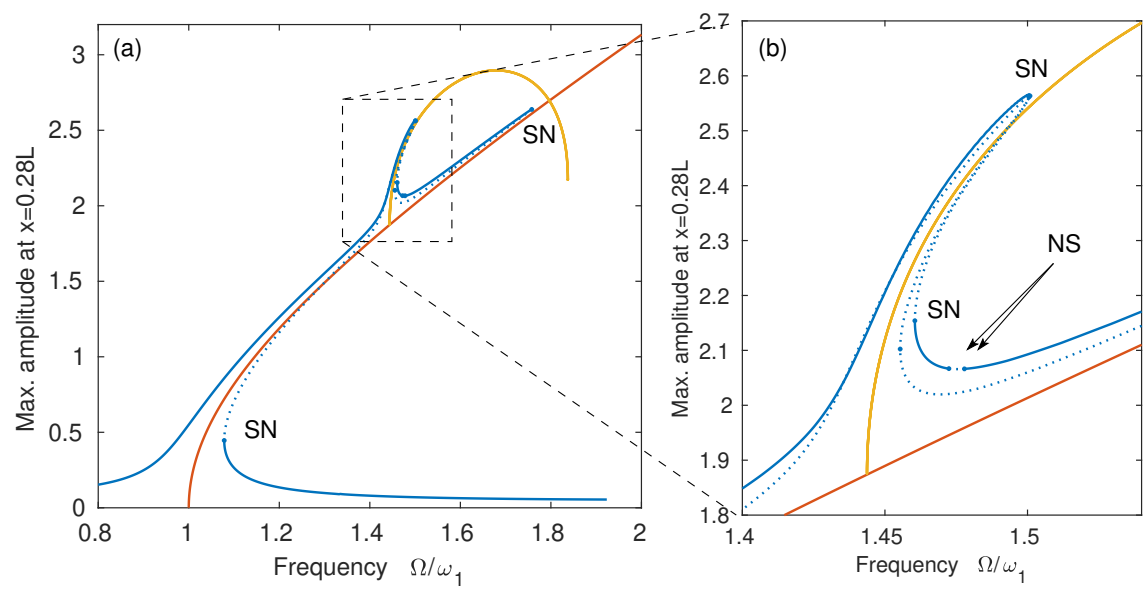

Figure 14: Forced response (solid blue line) of the hinged-hinged beam driven with point forces $P_{1}(t)$ and $P_{2}(t)$. Maximum amplitude of the transverse displacement, over one period, at $x_{\text {disp }}=0.28 L$. The backbones of the first mode and the 1:3 internal resonance are also shown in orange and yellow.

To further validate and test our stability computation algorithm, the forced case considered in [Lew97, LTD12] is also analyzed. The beam is driven by two out of phase point forces $P_{1}(t)=-13.63 E I r / L^{3} \cos \Omega t$ and $P_{2}(t)=9.62 E I r / L^{3} \cos \Omega t$, respectively imposed at $x_{1}=L / 4$ and $x_{2}=3 L / 4$. The damping ratios are adjusted to have a mass proportional damping, such that $\xi_{k}=0.005 \omega_{1} / \omega_{k}$. The maximum amplitude of the transverse displacement of the beam at $x_{\text {disp }}=0.28 \mathrm{~L}$ is shown in Fig. 14 . It has the overall shape of the forced response of a hardening Duffing oscillator, with an additional resonance around $\Omega=1.5 \omega_{1}$ due to the 1:3 internal resonance with mode 2 . A number of saddle node bifurcations are obtained, as well as two Neimarck-Sacker bifurcations, already observed in [LTD12].

Then, the same process is applied with similar forces, with an amplitude increased by a factor $4: 4 P_{1}$ and $4 P_{2}$, to observe the 1:2 internal resonance. The blue curve of Fig. 15 is obtained. The same qualitative behaviour is obtained, with losses of stability due to saddle-node and Neimark-Sacker bifurcation at low amplitude. At higher amplitude, around $\Omega=3 \omega_{1}$, the 1:2 internal resonance is observed with four additional resonances obtained after subcritical and supercritical pitchfork (symmetry breaking) bifurcations. 

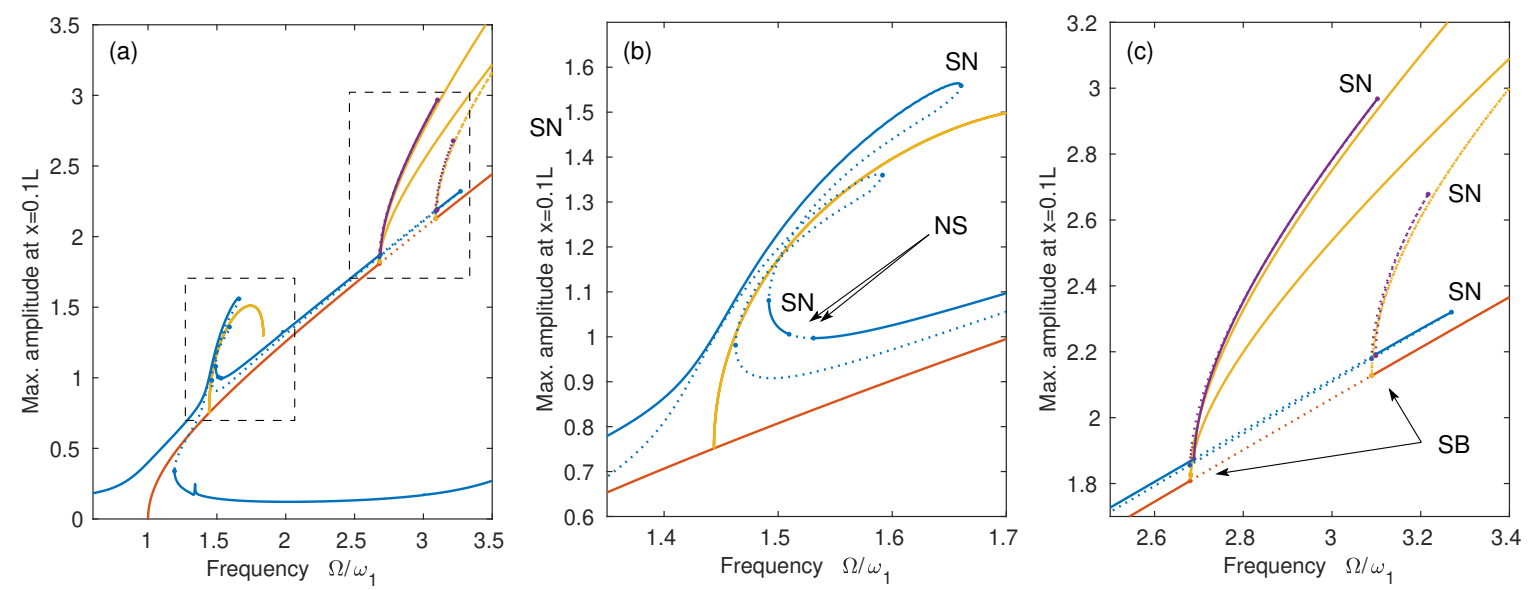

Figure 15: Forced response (solid blue line) of the hinged-hinged beam driven with point forces $4 P_{1}(t)$ and $4 P_{2}(t)$. Maximum amplitude of the transverse displacement, over one period, at $x_{\text {disp }}=0.1 L$. The backbones of the first mode, and the 1:3 and 1:2 internal resonances are also shown in orange and yellow.

\section{Conclusion and perspectives}

With the use of condensation variables to recast a set of smooth nonlinear ODEs in a quadratic format, not only it becomes relatively straightforward to compute and follow periodic solutions through harmonic balance formulation and continuation techniques, but Hill's matrix emerging from Floquet theory is computed automatically. One could highlight two immediate advantages from this outcome. Firstly, the efficiency of the stability and bifurcation analysis of periodic orbits of nonlinear dynamical systems is now greatly enhanced when using harmonic balance and asymptotic numerical methods. Secondly, it becomes natural to decompose the perturbation around the studied periodic stationary state in its orthogonal basis of Floquet forms since the latter results directly from the spectrum of Hill matrix. Alike normal modes for the perturbations of fixed points of nonlinear ODEs, FFs give physical insights in the instability mechanism of a periodic orbit and provide notably directions of bifurcation. The present paper brings new convincing arguments for the eventual use of nonlinear algorithms in the frequency domain to compute periodic solutions of ODEs. It notably highlights the fact that alike Monodromy matrices and their Floquet multipliers for orthogonal collocation or shooting techniques in the time domain, Hill's matrices and their Floquet exponents could be constructed as a by-product of Harmonic balance methods. In future works, we will strive to expand our results to larger class of ordinary differential systems such as Differential Algebraic Equations (DAE). Also, another theoretical challenge concerns the improvement of the computation of the spectrum of Hill's matrix that currently could require the calculation of eigenproblems with large size.

\section{Bibliography}

[ACV05] Remi Arquier, Bruno Cochelin, and Christophe Vergez. Manlab-logiciel de continuation intéractif. In 7éme colloque national en calcul des structures (France, Giens). nc, 2005.

[AP98] Uri M Ascher and Linda R Petzold. Computer methods for ordinary differential equations and differential-algebraic equations, volume 61. Siam, 1998.

[BL18] Barend Bentvelsen and Arnaud Lazarus. Modal and stability analysis of structures in periodic elastic states: application to the ziegler column. Nonlinear Dynamics, 91(2):1349-1370, 2018. 
[BM90] Peter J Bryant and John W Miles. On a periodically forced, weakly damped pendulum. part 3: Vertical forcing. The ANZIAM Journal, 32(1):42-60, 1990.

[CDPF94a] Bruno Cochelin, Noureddine Damil, and Michel Potier-Ferry. Asymptotic-numerical methods and pade approximants for non-linear elastic structures. International journal for numerical methods in engineering, 37(7):1187-1213, 1994.

[CDPF94b] Bruno Cochelin, Noureddine Damil, and Michel Potier-Ferry. The asymptotic-numerical method: an efficient perturbation technique for nonlinear structural mechanics. Revue européenne des éléments finis, 3(2):281-297, 1994.

[CM13] Bruno Cochelin and Marc Medale. Power series analysis as a major breakthrough to improve the efficiency of asymptotic numerical method in the vicinity of bifurcations. Journal of Computational Physics, 236:594-607, 2013.

[Coc94] Bruno Cochelin. A path-following technique via an asymptotic-numerical method. Computers \& structures, 53(5):1181-1192, 1994.

[CV09] Bruno Cochelin and Christophe Vergez. A high order purely frequency-based harmonic balance formulation for continuation of periodic solutions. Journal of sound and vibration, 324(1):243-262, 2009.

[CW84] Robert A Calico and William E Wiesel. Control of time-periodic systems. Journal of Guidance, Control, and Dynamics, 7(6):671-676, 1984.

[DKK91] Eusebius Doedel, Herbert B Keller, and Jean Pierre Kernevez. Numerical analysis and control of bifurcation problems (i): bifurcation in finite dimensions. International journal of bifurcation and chaos, 1(03):493-520, 1991.

[Doe81] Eusebius J Doedel. Auto: A program for the automatic bifurcation analysis of autonomous systems. Congr. Numer, 30:265-284, 1981.

[DRMK15] Thibaut Detroux, Ludovic Renson, Luc Masset, and Gaëtan Kerschen. The harmonic balance method for bifurcation analysis of large-scale nonlinear mechanical systems. Computer Methods in Applied Mechanics and Engineering, 296:18-38, 2015.

[Flo79] Gaston Floquet. Sur la théorie des équations différentielles linéaires. In Annales Scientifiques de l'Ecole Normale Superieure, volume 8, pages 3-132, 1879.

[GCV19] Louis Guillot, Bruno Cochelin, and Christophe Vergez. A generic and efficient Taylor series based continuation method using a quadratic recast of smooth nonlinear systems. International Journal of Numerical Methods in Engineering, 119(4):261-280, 2019.

[GGHW00] R.E. Goldstein, A. Goriely, G. Huber, and C.W. Wolgemuth. Bistable helices. Physical review letters, 84(7):1631-1634, 2000.

[GGTD19] A. Givois, A. Grolet, O. Thomas, and J.-F. Deü. On the frequency response computation of geometrically nonlinear flat structures using reduced-order finite element models. Nonlinear Dynamics, $97(2): 1147-1781,2019$.

[GH83a] J. Guckenheimer and P. Holmes. Nonlinear oscillations, dynamical systems and bifurcations of vector fields. Springer-Verlag, New-York, 1983.

[GH83b] John Guckenheimer and Philip Holmes. Nonlinear oscillations, dynamical systems, and bifurcations of vector fields, volume 42. New York Springer Verlag, 1983.

[Goo51] Richard M Goodwin. The nonlinear accelerator and the persistence of business cycles. Econometrica: Journal of the Econometric Society, pages 1-17, 1951.

[GVC18] Louis Guillot, Christophe Vergez, and Bruno Cochelin. Continuation of periodic solutions of various types of Delay Differential Equations using Asymptotic Numerical Method and Harmonic Balance Method. working paper or preprint, November 2018.

[GVVC17] Louis Guillot, Pierre Vigué, Christophe Vergez, and Bruno Cochelin. Continuation of quasi-periodic solutions with two-frequency harmonic balance method. Journal of Sound and Vibration, 394:434-450, 2017.

[Hil86] George William Hill. On the part of the motion of the lunar perigee which is a function of the mean motions of the sun and moon. Acta mathematica, 8(1):1-36, 1886

$\left[\mathrm{KAL}^{+}{ }^{10}\right] \quad \mathrm{S}$ Karkar, R Arquier, A Lazarus, O Thomas, C Vergez, and B Cochelin. Manlab: an interactive path-following and bifurcation analysis software, 2010.

[KB16] Nikolai Mitrofanovich Krylov and Nikolai Nikolaevich Bogoliubov. Introduction to Non-Linear Mechanics.(AM-11), volume 11. Princeton University Press, 2016. 
[KCV13] Sami Karkar, Bruno Cochelin, and Christophe Vergez. A high-order, purely frequency based harmonic balance formulation for continuation of periodic solutions: The case of non-polynomial nonlinearities. Journal of Sound and Vibration, 332(4):968-977, 2013.

[KCV14] Sami Karkar, Bruno Cochelin, and Christophe Vergez. A comparative study of the harmonic balance method and the orthogonal collocation method on stiff nonlinear systems. Journal of Sound and Vibration, 333(12):2554-2567, 2014.

[Kel87] HB Keller. Lectures on numerical methods in bifurcation problems. Applied Mathematics, 217:50, 1987.

[Kha96] Hassan K Khalil. Noninear systems. Prentice-Hall, New Jersey, 2(5):5-1, 1996.

$\left[\mathrm{KHP}^{+}{ }^{09]}\right.$ Najib Kacem, Sebastien Hentz, David Pinto, Bruno Reig, and V Nguyen. Nonlinear dynamics of nanomechanical beam resonators: improving the performance of nems-based sensors. Nanotechnology, 20(27):275501, 2009.

[KPGV09] G. Kerschen, M. Peeters, J.-C. Golinval, and A. F. Vakakis. Nonlinear normal modes, parti: A useful framework for the structural dynamicist. Mechanical Systems and Signal Processing, 23:170-194, 2009.

[KVC12] Sami Karkar, Christophe Vergez, and Bruno Cochelin. Oscillation threshold of a clarinet model: A numerical continuation approach. The Journal of the Acoustical Society of America, 131(1):698-707, 2012.

[Laz19] Arnaud Lazarus. Discrete dynamical stabilization of a naturally diverging mass in a harmonically time-varying potential. Physica D: Nonlinear Phenomena, 386:1-7, 2019.

[Lew94a] R. Lewandowski. Non-linear free vibrations of beams by the finite element and continuation methods. Journal of Sound and Vibration, 170(5):577-593, 1994.

[Lew94b] R. Lewandowski. Solutions with bifurcation points for free vibration of beams: An analytical approach. Journal of Sound and Vibration, 177(2):239-249, 1994.

[Lew97] R Lewandowski. Computational formulation for periodic vibration of geometrically nonlinear structures-part 2: numerical strategy and examples. International journal of solids and structures, 34(15):1949-1964, 1997.

[LT10] Arnaud Lazarus and Olivier Thomas. A harmonic-based method for computing the stability of periodic solutions of dynamical systems. Comptes Rendus Mécanique, 338(9):510-517, 2010.

[LTD12] A. Lazarus, O. Thomas, and J.-F. Deü. Finite elements reduced order models for nonlinear vibrations of stratified piezoelectric beams with applications to nems. Finite Elements in Analysis and Design, 49:35-51, 2012.

$\left[\mathrm{MAFG}^{+}\right.$03] Francisco J Munoz-Almaraz, Emilio Freire, J Galán, E Doedel, and André Vanderbauwhede. Continuation of periodic orbits in conservative and hamiltonian systems. Physica D: Nonlinear Phenomena, 181(1-2):1-38, 2003.

[man] Manlab - an interactive path-following and bifurcation analysis software. available at https://manlab. lma.cnrs-mrs.fr.

[Moo05] Gerald Moore. Floquet theory as a computational tool. SIAM journal on numerical analysis, $42(6): 2522-2568,2005$.

[MTT15] M. Monteil, O. Thomas, and C. Touzé. Identification of mode couplings in nonlinear vibrations of the steelpan. Applied Acoustics, 89:1-15, 2015. doi: 10.1016/j.apacoust.2014.08.008.

[MTTB14] M. Monteil, C. Touzé, O. Thomas, and S. Benacchio. Nonlinear forced vibrations of thin structures with tuned eigenfrequencies: the cases of 1:2:4 and 1:2:2 internal resonances. Nonlinear Dynamics, 75(1-2):175-200, 2014. doi: 10.1007/s11071-013-1057-7.

[Nay00] A. H. Nayfeh. Nonlinear interactions: analytical, computational and experimental methods. Wiley series in nonlinear science, New-York, 2000.

[NB89] A. H. Nayfeh and B. Balachandran. Modal interactions in dynamical and structural systems. ASME Applied Mech. Rev., 42(11):175-201, 1989.

[NB95] A. H. Nayfeh and B. Balachandran. Applied Nonlinear Dynamics. Analytical, computational and experimental Methods. Wiley, 1995.

[NM79] A. H. Nayfeh and D. T. Mook. Nonlinear oscillations. John Wiley \& sons, inc., New-York, 1979.

[PBTJR13] Loïc Peletan, Sébastien Baguet, Mohamed Torkhani, and Georges Jacquet-Richardet. A comparison of stability computational methods for periodic solution of nonlinear problems with application to rotordynamics. Nonlinear Dynamics, 72(3):1-12, 2013. 
[PF79] M. Potier-Ferry. Perturbed bifurcation theory. Journal of Differential Equations, 33:112-146, 1979.

[PVS ${ }^{+}$09] Maxime Peeters, Régis Viguié, Guillaume Sérandour, Gaëtan Kerschen, and J-C Golinval. Nonlinear normal modes, part ii: Toward a practical computation using numerical continuation techniques. Mechanical systems and signal processing, 23(1):195-216, 2009.

[Ric12] John A Richards. Analysis of periodically time-varying systems. Springer Science \& Business Media, 2012 .

[RKC16] L. Renson, G. Kerschen, and B. Cochelin. Numerical computation of nonlinear normal modes in mechanical engineering. Journal of Sound and Vibration, 364:177-206, 2016.

[Sey88] Rüdiger Seydel. From equilibrium to chaos: practical bifurcation and stability analysis. North-Holland, 1988.

[SP93] S. Shaw and C. Pierre. Normal modes for nonlinear vibratory systems. J. Sound. Vib., 164(1):85-124, 1993.

[Str01] Steven H Strogatz. Nonlinear dynamics and chaos: with applications to physics, biology and chemistry. Perseus publishing, 2001.

[TLT10] Olivier Thomas, Arnaud Lazarus, and Cyril Touzé. A harmonic-based method for computing the stability of periodic oscillations of non-linear structural systems. In ASME 2010 International Design Engineering Technical Conferences and Computers and Information in Engineering Conference, pages 883-892. American Society of Mechanical Engineers, 2010.

[TSD16] O. Thomas, A. Sénéchal, and J. F. Deü. Hardening / softening behaviour and reduced order modelling of nonlinear vibrations of rotating cantilever beams. Nonlinear dynamics, 86(2):1293-1318, 2016.

[TTC04] C. Touzé, O. Thomas, and A. Chaigne. Hardening/softening behaviour in non-linear oscillations of structural systems using non-linear normal modes. Journal of Sound Vibration, 273(1-2):77-101, 2004.

[TTC05] O. Thomas, C. Touzé, and A. Chaigne. Non-linear vibrations of free-edge thin spherical shells: modal interaction rules and 1:1:2 internal resonance. International Journal of Solids and Structures, 42(1112):3339-3373, 2005 .

[TTL07] O. Thomas, C. Touzé, and É. Luminais. Non-linear vibrations of free-edge thin spherical shells: experiments on a 1:1:2 internal resonance. Nonlinear Dynamics, 49(1-2):259-284, 2007.

[TVK00] Tero Tolonen, V Valimaki, and Matti Karjalainen. Modeling of tension modulation nonlinearity in plucked strings. IEEE Transactions on Speech and Audio Processing, 8(3):300-310, 2000.

[Ura65] Minoru Urabe. Galerkin's procedure for nonlinear periodic systems. Archive for Rational Mechanics and Analysis, 20(2):120-152, 1965.

[WH01] Xiaodong Wang and Jack K Hale. On monodromy matrix computation. Computer methods in applied mechanics and engineering, 190(18):2263-2275, 2001.

[WK50] S. Woinowsky-Krieger. The effect of axial force on the vibration of hinged bars. Journal of Applied Mechanics, 17:35-36, 1950.

[WW96] Edmund Taylor Whittaker and George Neville Watson. A course of modern analysis. Cambridge university press, 1996.

[ZHA04] Jun Zhou, Tomomichi Hagiwara, and Mituhiko Araki. Spectral characteristics and eigenvalues computation of the harmonic state operators in continuous-time periodic systems. Systems E control letters, 53(2):141-155, 2004 